\title{
Pacific
}

Journal of

Mathematics

\section{SINGULARITIES OF FREE GROUP CHARACTER VARIETIES}

Carlos Florentino and Sean Lawton 


\title{
SINGULARITIES OF FREE GROUP CHARACTER VARIETIES
}

\author{
Carlos Florentino And Sean Lawton
}

\begin{abstract}
Let $\mathfrak{X}_{r}$ be the moduli space of $\mathrm{SL}_{n}, \mathrm{SU}_{n}, \mathrm{GL}_{n}$, or $\mathrm{U}_{n}$-valued representations of a rank $r$ free group. We classify the algebraic singular stratification of $\mathfrak{X}_{r}$. This comes down to showing that the singular locus corresponds exactly to reducible representations if there exist singularities at all. Then by relating algebraic singularities to topological singularities, we show the moduli spaces $\mathfrak{X}_{r}$ generally are not topological manifolds, except for a few examples we explicitly describe.
\end{abstract}

\section{Introduction}

During the last few decades, character varieties have played important roles in knot theory, hyperbolic geometry, Higgs and vector bundle theory, and quantum field theory. However, many of their fundamental properties and structure are not completely understood.

In this article, we first classify the (algebraic) singular locus of $\mathrm{SL}_{n}$ and $\mathrm{GL}_{n^{-}}$ character varieties of free groups by relating the existence of a singularity with the reducibility of the corresponding representation. We then classify all such character varieties that arise as manifolds by explicitly describing the topological neighborhoods of generic singularities. The results we obtain do not necessarily extend to general $G$-character varieties of finitely generated groups $\Gamma$, if $G$ is not one of $\mathrm{SL}_{n}, \mathrm{SU}_{n}, \mathrm{GL}_{n}$, or $\mathrm{U}_{n}$ and $\Gamma$ is not free; explicit counterexamples can be obtained via methods different from those considered in this paper (see Section 3I). Our first main theorem generalizes results in [Heusener and Porti 2004], and our second main theorem generalizes results in [Bratholdt and Cooper 2001]. They may be described more precisely as follows.

Let $\mathrm{F}_{r}$ be a rank $r$ free group and let $G$ be a reductive complex algebraic group with $K$ a maximal compact subgroup (see Section 2). Let $\mathfrak{R}_{r}(G)=\operatorname{Hom}\left(\mathrm{F}_{r}, G\right)$ and $\mathfrak{R}_{r}(K)=\operatorname{Hom}\left(\mathrm{F}_{r}, K\right)$ be varieties of representations, and let $G$, respectively

This work was partially supported by the Center for Mathematical Analysis, Geometry and Dynamical Systems at I.S.T., and by the Fundação para a Ciência e a Tecnologia through the programs Praxis XXI, POCI/ MAT/ 58549/ 2004 and FEDER..

MSC2010: primary 14B05, 32S05; secondary 14L30, 20CXX, $14 \mathrm{P} 10$.

Keywords: character variety, singularities, local topology. 
$K$, act by conjugation on these representation spaces.

Consider the space $\mathfrak{X}_{r}(K):=\mathfrak{R}_{r}(K) / K$ which is the conjugation orbit space of $\mathfrak{R}_{r}(K)$ where $\rho \sim \psi$ if and only if there exists $k \in K$ so $\rho=k \psi k^{-1}$. Let $\mathbb{C}\left[\mathfrak{R}_{r}(G)\right]$ be the affine coordinate ring of $\mathfrak{R}_{r}(G)$ and let $\mathbb{C}\left[\mathfrak{R}_{r}(G)\right]^{G}$ be the subring of $G$ conjugation invariants. Then define

$$
\mathfrak{X}_{r}(G):=\operatorname{Spec}_{\text {max }}\left(\mathbb{C}\left[\Re_{r}(G)\right]^{G}\right),
$$

which parametrizes unions of conjugation orbits where two orbits are in the same union if and only if their closures have a nonempty intersection.

The space $\mathfrak{X}_{r}(G)$, called the $G$-character variety of $\mathrm{F}_{r}$, is a complex affine variety and so has a well-defined (algebraic) singular locus (a proper subvariety) which we denote by $\mathfrak{X}_{r}(G)^{\text {sing }}$. Similarly, $\mathfrak{X}_{r}(K)$ is a semialgebraic set and so has a real algebraic coordinate ring which likewise determines an algebraic singular locus $\mathfrak{X}_{r}(K)^{\text {sing }}$. For simplicity, despite the fact it is generally not an algebraic set, we will also refer to $\mathfrak{X}_{r}(K)$ as a character variety.

We will be mainly concerned with the cases when $G$ is the general linear group $\mathrm{GL}_{n}$ or the special linear group $\mathrm{SL}_{n}$ (over $\mathbb{C}$ ), for which $K$ is the unitary group $\mathrm{U}_{n}$ or the special unitary group $\mathrm{SU}_{n}$, respectively. In these cases a representation $\rho$ is called irreducible if with respect to the standard action of $G$, respectively $K$, on $\mathbb{C}^{n}$ the induced action of $\rho\left(\mathrm{F}_{r}\right)$ does not have any nontrivial proper invariant subspaces. Otherwise $\rho$ is called reducible. This allows one to define the sets $\mathfrak{X}_{r}(G)^{\text {red }}$ and $\mathfrak{X}_{r}(K)^{\text {red }}$ which correspond to the spaces of equivalence classes in $\mathfrak{X}_{r}(G)$, respectively $\mathfrak{X}_{r}(K)$, that have a representative which is reducible.

In Section 2, we show that the (algebraic) singular locus of $\mathfrak{X}_{r}\left(\mathrm{SL}_{n}\right)$ and $\mathfrak{X}_{r}\left(\mathrm{GL}_{n}\right)$ respectively determines the (algebraic) singular locus of $\mathfrak{X}_{r}\left(\mathrm{SU}_{n}\right)$ and $\mathfrak{X}_{r}\left(\mathrm{U}_{n}\right)$. We then show $\mathfrak{X}_{r}\left(\mathrm{SL}_{n}\right) \subset \mathfrak{X}_{r}\left(\mathrm{GL}_{n}\right)$ has its singular locus determined by the singular locus of $\mathfrak{X}_{r}\left(\mathrm{GL}_{n}\right)$. This reduces the classification of the singular loci of these four families of moduli spaces to $\mathfrak{X}_{r}\left(\mathrm{GL}_{n}\right)$ alone. We end Section 2 with examples of $\mathfrak{X}_{r}(G)$ that are homeomorphic to manifolds with boundary; we conjectured in [Florentino and Lawton 2009] that these were the only examples.

It is straightforward to establish that $\mathfrak{X}_{1}\left(\mathrm{SL}_{n}\right) \cong \mathbb{C}^{n-1}$ and $\mathfrak{X}_{2}\left(\mathrm{SL}_{2}\right) \cong \mathbb{C}^{3}$ are affine spaces and so smooth, and $\mathfrak{X}_{1}\left(\mathrm{SL}_{n}\right)^{\text {red }}=\mathfrak{X}_{1}\left(\mathrm{SL}_{n}\right)$. In [Heusener and Porti 2004], it is shown that $\mathfrak{X}_{r}\left(\mathrm{SL}_{2}\right)^{\text {sing }}=\mathfrak{X}_{r}\left(\mathrm{SL}_{2}\right)^{\text {red }}$ for $r \geq 3$. More generally, one can establish that all irreducible representations in $\mathrm{SL}_{n}$-character varieties of free groups are in fact smooth; that is $\mathfrak{X}_{r}\left(\mathrm{SL}_{n}\right)^{\text {sing }} \subset \mathfrak{X}_{r}\left(\mathrm{SL}_{n}\right)^{\text {red }}$. In [Lawton 2007] it is shown that the singular locus of $\mathfrak{X}_{2}\left(\mathrm{SL}_{3}\right)$ corresponds exactly to the set of equivalence classes of reducible representations; that is, $\mathfrak{X}_{2}\left(\mathrm{SL}_{3}\right)^{\text {red }}=\mathfrak{X}_{2}\left(\mathrm{SL}_{3}\right)^{\text {sing }}$. These examples generalize to our first main result:

Theorem 1.1. Let $r, n \geq 2$. Let $G$ be $\mathrm{SL}_{n}$ or $\mathrm{GL}_{n}$ and $K$ be $\mathrm{SU}_{n}$ or $\mathrm{U}_{n}$. Then $\mathfrak{X}_{r}(G)^{\text {red }}=\mathfrak{X}_{r}(G)^{\text {sing }}$ and $\mathfrak{X}_{r}(K)^{\text {red }}=\mathfrak{X}_{r}(K)^{\text {sing }}$ if and only if $(r, n) \neq(2,2)$. 
In fact we are able to use an induction argument to completely classify the singular stratification of these semialgebraic spaces. The proof and development of this result constitutes Section 3, including a brief review of a weak version of the celebrated Luna slice theorem.

Theorem 1.1 is sharper than it might appear at first. Replacing $\mathrm{F}_{r}$ by a general finitely presented group $\Gamma$ one can find examples where irreducibles are singular and examples where reducibles are smooth. On the other hand, changing $G$ to a general reductive complex algebraic group, we find there are examples where irreducibles are singular. In Section 3I, we discuss this in further detail.

A locally Euclidean Hausdorff space $M$ with a countable basis is called a topological manifold. More generally, if the neighborhoods are permitted to be Euclidean half-spaces then $M$ is said to be a topological manifold with boundary. In [Florentino and Lawton 2009] we determined the homeomorphism type of $\mathfrak{X}_{r}\left(\mathrm{SU}_{n}\right)$ in the cases $(r, n)=(r, 1),(1, n),(2,2),(2,3)$, and $(3,2)$ where we showed all were topological manifolds with boundary; this is reviewed in Section 2B. In [Bratholdt and Cooper 2001] it is established that the $\mathfrak{X}_{r}\left(\mathrm{SU}_{2}\right)$ are not topological manifolds when $r \geq 4$.

Motivated by this we conjectured in [Florentino and Lawton 2009] that the examples computed there are the only cases where a topological manifold with boundary arise. Our second main theorem in this paper establishes that conjecture:

Theorem 1.2. Let $r, n \geq 2$. Let $G$ be $\mathrm{SL}_{n}$ or $\mathrm{GL}_{n}$ and $K$ be $\mathrm{SU}_{n}$ or $\mathrm{U}_{n}$. $\mathfrak{X}_{r}(G)$ is a topological manifold with boundary if and only if $(r, n)=(2,2) . \mathfrak{X}_{r}(K)$ is a topological manifold with boundary if and only if $(r, n)=(2,2),(2,3)$, or $(3,2)$.

Theorem 1.1 and the observation that the reducible locus is nonempty for $n \geq 2$, does not immediately imply Theorem 1.2 since algebraic singularities may or may not be an obstruction to the existence of a Euclidean neighborhood (topological singularities). For example, both the varieties given by $x y=0$ and $y^{2}=x^{3}$ in $\mathbb{C}^{2}$ (or $\mathbb{R}^{2}$ ) are (algebraically) singular at the point $(0,0)$ but only the latter has a Euclidean neighborhood at the origin. So, only the former is topologically singular. The variety $x y=0$ is reducible; an example of an irreducible variety that has an algebraic singularity that is also a topological singularity is the affine cone over $\mathbb{C P} \times \mathbb{C P}^{1}$ discussed in Section 3E.

The proof of Theorem 1.2 constitutes Section 4. To prove our main theorems we use slice theorems and explicitly describe the homeomorphism type of neighborhoods (showing them to be non-Euclidean) for a family of examples. It is interesting to note that since $\mathfrak{X}_{r}\left(\mathrm{SL}_{n}\right)$ deformation retracts to $\mathfrak{X}_{r}\left(\mathrm{SU}_{n}\right)$, by [Florentino and Lawton 2009], it must be the case that for $(r, n)=(2,3)$ and $(3,2)$ the non-Euclidean neighborhoods deformation retract to Euclidean neighborhoods. Curiously, these are the only cases $(n \geq 2)$ where $\mathfrak{X}_{r}\left(\mathrm{SU}_{n}\right)$ is a topological manifold, and both are homeomorphic to spheres (see [Florentino and Lawton 2009] or Section 2B). 


\section{Character varieties}

Let $G$ be a complex affine reductive algebraic group and let $K$ be a maximal compact subgroup. Then, $G=K_{\mathbb{C}}$ is the complexification of $K$ (the set complex zeros of $K$ as a real algebraic set). For instance, $K_{\mathbb{C}}=\mathrm{SL}_{n}$ is the complexification of $K=\mathrm{SU}_{n}$, and $K_{\mathbb{C}}=\mathrm{GL}_{n}$ is the complexification of $K=\mathrm{U}_{n}$.

Let $\Gamma$ be a finitely generated group and let $\Re_{\Gamma}(G)=\operatorname{Hom}(\Gamma, G)$ be the $G$-valued representations of $\Gamma$. We call $\mathfrak{R}_{\Gamma}(G)$ the $G$-representation variety of $\Gamma$, although it is generally only an affine algebraic set.

In the category of affine varieties, $\mathfrak{R}_{\Gamma}(G)$ has a quotient by the conjugation action of $G$, a regular action, given by $\rho \mapsto g \rho g^{-1}$. This quotient is realized as $\mathfrak{X}_{\Gamma}(G)=$ $\operatorname{Spec}_{\max }\left(\mathbb{C}\left[\Re_{\Gamma}(G)\right]^{G}\right)$, where $\mathbb{C}\left[\mathfrak{R}_{\Gamma}(G)\right]^{G}$ is the subring of invariant polynomials in the affine coordinate ring $\mathbb{C}\left[\mathfrak{R}_{\Gamma}(G)\right]$. We call $\mathfrak{X}_{\Gamma}(G)$ the $G$-character variety of $\Gamma$. Concretely, it parametrizes unions of conjugation orbits where two orbits are in the same union if and only if their closures intersect nontrivially. Within each union of orbits, denoted $[\rho]$ and called an extended orbit equivalence class, there is a unique closed orbit (having minimal dimension). Any representative from this closed orbit is called a polystable point. For $\mathrm{SL}_{n}$ and $\mathrm{GL}_{n}$ the polystable points will have the property that with respect to the action of $\rho(\Gamma)$ on $\mathbb{C}^{n}$, they are completely reducible; that is, each decomposes into a finite direct sum of irreducible subactions (on nonzero subspaces).

Let $\mathrm{F}_{r}=\left\langle x_{1}, \ldots, x_{r}\right\rangle$ be a rank $r$ free group. The $G$-representation variety of $\mathrm{F}_{r}$, and the $G$-character variety of $\mathrm{F}_{r}$ will simply be denoted by $\mathfrak{R}_{r}(G)$ and $\mathfrak{X}_{r}(G)$, respectively. The evaluation mapping $\mathfrak{R}_{r}(G) \rightarrow G^{r}$ defined by sending $\rho \mapsto\left(\rho\left(x_{1}\right), \ldots, \rho\left(x_{r}\right)\right)$ is a bijection and since $G$ is a smooth affine variety, $\Re_{r}(G)$ naturally inherits the structure of a smooth affine variety as well. Note that we are not assuming that an algebraic variety is irreducible. Whenever $G$ is an irreducible algebraic set however, $\mathfrak{R}_{r}(G)$ is irreducible, and consequently $\mathfrak{X}_{r}(G)$ is irreducible as well.

Since an algebraic reductive group over $\mathbb{C}$ is always linear, we can assume that $G$ is a subgroup of $\mathrm{GL}_{N}$, for some $N$, and hence $\mathfrak{R}_{r}(G) \subset \mathbb{C}^{r N^{2}}$. So, $\mathfrak{R}_{r}(G)$ inherits the ball topology. Given a set of generators $f_{1}, \ldots, f_{k}$ of the ring of invariants $\mathbb{C}\left[\mathfrak{R}_{r}(G)\right]^{G}, \mathfrak{X}_{r}(G)$ also inherits the ball topology from the embedding of $\mathfrak{X}_{r}(G)$ into $\mathbb{C}^{k}$ given by $[\rho] \mapsto\left(f_{1}(\rho), \ldots, f_{k}(\rho)\right)$. In this topology $\mathfrak{X}_{r}(G)$ is Hausdorff and has a countable basis. Although the ball topology is dependent on an embedding a priori, an affine embedding corresponds exactly to a set of generators for the associated ring, but all choices result in the same homeomorphism type, so the ball topology is intrinsic. Also, in the ball topology, at each point in $\mathfrak{X}_{r}(G)$ there is a neighborhood homeomorphic to a real cone over a space with Euler characteristic 0 [Sullivan 1971]. 
Given a compact Lie group $K$, for brevity we also call the orbit space $\mathfrak{X}_{r}(K)=$ $\mathfrak{R}_{r}(K) / K$ a $K$-character variety of $\mathrm{F}_{r}$. Note however that $\mathfrak{X}_{r}(K)$ is generally only a semialgebraic set, and does not equal, in general, the set of real points of a complex variety. In this case, the topology, also Hausdorff with a countable basis, is the quotient topology. $\mathfrak{X}_{r}(K)$ is compact since $K$ is compact. Likewise, it is path-connected whenever $K$ is path-connected.

Definition 2.1. Let $\rho: \Gamma \rightarrow G$ be a representation into a reductive complex algebraic group. If the image of $\rho$ does not lie in a parabolic subgroup of $G$, then $\rho$ is called irreducible. If, for every parabolic $P$ containing $\rho(\Gamma)$ there is a Levi factor $L \subset P$ such that $\rho(\Gamma) \subset L$, then $\rho$ is called completely reducible.

For $\mathrm{SL}_{n}$ and $\mathrm{GL}_{n}$ the irreducible representations are exactly those that, with respect to their actions on $\mathbb{C}^{n}$, do not admit any proper (nontrivial) invariant subspaces. Any representation that is not irreducible is called reducible. Denote the set of reducible representations by $\mathfrak{R}_{\Gamma}(G)^{\text {red }}$. A point is called stable if the stabilizer is finite and if the orbit is closed.

The following theorem can be found in [Sikora 2012], building on earlier work in [Johnson and Millson 1987, pp. 54-57]. Let $\mathrm{P} G=G / Z(G)$ where $Z(G)$ is the center. Note that the action of P $G$ and $G$ define the same GIT quotients and the same orbit spaces and thus, since the $\mathrm{P} G$ action is effective, we will sometimes consider this action.

Theorem 2.2 [Johnson and Millson 1987; Sikora 2012]. Let $G$ be reductive. The irreducibles are exactly the stable points under the action of $\mathrm{PG}$ on $\mathfrak{R}_{\Gamma}(G)$. The completely reducibles are the polystable points.

Definition 2.3. The reducibles $\mathfrak{X}_{\Gamma}(G)^{\text {red }}$ are the image of the projection

$$
\mathfrak{R}_{\Gamma}(G)^{\mathrm{red}} \subset \mathfrak{R}_{\Gamma}(G) \longrightarrow \mathfrak{X}_{\Gamma}(G) .
$$

Since $\mathfrak{R}_{r}(G) \cong G^{r}$ all points are smooth, and since $\mathfrak{X}_{r}(G)$ is an affine quotient of a reductive group, there exists $\rho^{\mathrm{ss}} \in[\rho]$ which has a closed orbit and corresponds to a completely reducible representation. Thus, for $G$ either $\mathrm{SL}_{n}$ or $\mathrm{GL}_{n}$ we can assume it is in block diagonal form. In other words, $\rho^{\mathrm{ss}} \leftrightarrow\left(X_{1}, \ldots, X_{r}\right)$ where $X_{i}$ all have the same block diagonal form (if they are irreducible then there would be only one block). These representations induce a semisimple module structure on $\mathbb{C}^{n}$. We denote the set of semisimple representations by $\mathfrak{R}_{r}(G)^{\mathrm{ss}}$. We note that $\mathfrak{R}_{r}(G)^{\mathrm{ss}} / G \cong \mathfrak{X}_{r}(G)$ since all extended orbits have a semisimple representative, and that the semisimple representations are also the completely reducible representations which are also the polystable representations. Likewise, we denote the irreducible representations (those giving simple actions on $\mathbb{C}^{n}$ ) by $\mathfrak{R}_{r}(G)^{\mathrm{s}}$ and their quotient by $\mathfrak{X}_{r}(G)^{\mathrm{s}}$. 
2A. The determinant fibration. In order to compare $\mathrm{SL}_{n}$-character varieties to $\mathrm{GL}_{n}$ character varieties, the following setup will be useful. The usual exact sequence of groups given by the determinant of an invertible matrix

$$
\mathrm{SL}_{n} \rightarrow \mathrm{GL}_{n} \stackrel{\text { det }}{\rightarrow} \mathbb{C}^{*}
$$

induces (by fixing generators of $\mathrm{F}_{r}$, as before) what we will call the determinant map:

$$
\operatorname{det}: \mathfrak{X}_{r}\left(\mathrm{GL}_{n}\right) \rightarrow \operatorname{Hom}\left(\mathrm{F}_{r}, \mathbb{C}^{*}\right) \cong\left(\mathbb{C}^{*}\right)^{r}, \quad[\rho] \mapsto \operatorname{det}(\rho),
$$

where $\operatorname{det}(\rho)=\left(\operatorname{det}\left(X_{1}\right), \ldots, \operatorname{det}\left(X_{r}\right)\right)$, for $\rho=\left(X_{1}, \ldots, X_{r}\right) \in \mathfrak{R}_{r}\left(\mathrm{GL}_{n}\right)$. Note that the map is clearly well-defined on conjugation classes. Considering the algebraic torus $\left(\mathbb{C}^{*}\right)^{r}=\operatorname{Hom}\left(\mathrm{F}_{r}, \mathbb{C}^{*}\right)=\mathfrak{X}_{r}\left(\mathbb{C}^{*}\right)$ as an algebraic group (with identity $\mathbf{1}=$ $(1, \ldots, 1)$ and componentwise multiplication) it is immediate that the $\mathrm{SL}_{n}$-character variety is the "kernel" of the determinant map, $\mathfrak{X}_{r}\left(\mathrm{SL}_{n}\right)=\operatorname{det}^{-1}(\mathbf{1})$. Therefore, the sequence (1) induces another exact sequence

$$
\mathfrak{X}_{r}\left(\mathrm{SL}_{n}\right) \rightarrow \mathfrak{X}_{r}\left(\mathrm{GL}_{n}\right) \stackrel{\text { det }}{\rightarrow}\left(\mathbb{C}^{*}\right)^{r} .
$$

In this way, $\mathrm{SL}_{n}$-character varieties appear naturally as subvarieties of $\mathrm{GL}_{n}$-character varieties.

Note also that $\mathfrak{X}_{r}\left(\mathrm{GL}_{n}\right)$ can be viewed as a $\mathfrak{X}_{r}\left(\mathbb{C}^{*}\right)$-space, as it admits a welldefined action of this torus. That is, we can naturally define $\rho \cdot \lambda \in \mathfrak{X}_{r}\left(\mathrm{GL}_{n}\right)$, given $\rho \in \mathfrak{X}_{r}\left(\mathrm{GL}_{n}\right)$ and $\lambda \in \mathfrak{X}_{r}\left(\mathbb{C}^{*}\right)$. Given that $\mathrm{PSL}_{n}=\mathrm{GL}_{n} / / \mathbb{C}^{*}$, it is easy to see that the corresponding quotient is the $\mathrm{PSL}_{n}$-character variety:

$$
\mathfrak{X}_{r}\left(\mathrm{PSL}_{n}\right)=\mathfrak{X}_{r}\left(\mathrm{GL}_{n}\right) / / \mathfrak{X}_{r}\left(\mathbb{C}^{*}\right) .
$$

Also, $\mathrm{GL}_{n}^{r}$ is a quasiaffine subvariety of $\mathfrak{g l}(n, \mathbb{C})^{r}$. In fact, it is the principal open set defined by the product of the determinants of generic matrices. Since the determinant is an invariant function and taking invariants commutes with localizing at those invariants, we have

$$
\mathbb{C}\left[\mathrm{GL}_{n}^{r}\right]^{\mathrm{GL}_{n}} \approx \mathbb{C}\left[\mathfrak{g l}(n, \mathbb{C})^{r} / / \mathrm{GL}_{n}\right]\left[\frac{1}{\operatorname{det}\left(X_{1}\right) \cdots \operatorname{det}\left(X_{r}\right)}\right],
$$

where the expression on the right is the localization at the product of determinants.

We now prove how the fixed determinant character varieties, complex and compact, relate to the nonfixed determinant character varieties. Identify the cyclic group of order $n, \mathbb{Z}_{n}:=\mathbb{Z} / n \mathbb{Z}$, with $Z\left(\mathrm{SL}_{n}\right) \cong Z\left(\mathrm{SU}_{n}\right)$.

Theorem 2.4. The following are isomorphisms of semialgebraic sets:

(i) $\mathfrak{X}_{r}\left(\mathrm{GL}_{n}\right) \cong \mathfrak{X}_{r}\left(\mathrm{SL}_{n}\right) \times \mathfrak{X}_{r}\left(\mathbb{Z}_{n}\right) \mathfrak{X}_{r}\left(\mathrm{GL}_{1}\right)$.

(ii) $\mathfrak{X}_{r}\left(\mathrm{U}_{n}\right) \cong \mathfrak{X}_{r}\left(\mathrm{SU}_{n}\right) \times_{\mathfrak{X}_{r}\left(\mathbb{Z}_{n}\right)} \mathfrak{X}_{r}\left(\mathrm{U}_{1}\right)$. 
Proof. We first note that $\mathfrak{X}_{r}\left(\mathrm{U}_{1}\right) \cong\left(S^{1}\right)^{r}$ and $\mathfrak{X}_{r}\left(\mathrm{GL}_{1}\right) \cong\left(\mathbb{C}^{*}\right)^{r}$, and thus $\mathfrak{X}_{r}\left(\mathbb{Z}_{n}\right) \cong$ $\mathbb{Z}_{n}^{r}$, as the groups involved are abelian.

The determinant map (1) defines a principal $\mathrm{SL}_{n}$-bundle $\mathrm{SL}_{n} \hookrightarrow \mathrm{GL}_{n} \rightarrow \mathbb{C}^{*}$, which also expresses $\mathrm{GL}_{n} \cong \mathrm{SL}_{n} \rtimes \mathbb{C}^{*}$ as a semidirect product since there exists a homomorphic section ( $\mathrm{SL}_{n}$ is a normal subgroup).

Let $\mathbb{Z}_{n}$ correspond to $n$-th roots of unity $\omega_{k}=e^{\frac{2 \pi i k}{n}}$. As algebraic sets one can show directly, by the mapping $(A, \lambda) \mapsto \lambda A$, that $\mathrm{GL}_{n} \cong\left(\mathrm{SL}_{n} \times \mathbb{C}^{*}\right) / / \mathbb{Z}_{n}:=\mathrm{SL}_{n} \times \mathbb{Z}_{n} \mathbb{C}^{*}$ where $\mathbb{Z}_{n}$ acts by $\omega_{k} \cdot(g, \lambda)=\left(g \omega_{k}, \omega_{k}^{-1} \lambda\right)$ and $\mathbb{C}^{*}$ is the center of $\mathrm{GL}_{n}$. This implies that, as algebraic sets,

$$
\begin{aligned}
\mathfrak{X}_{r}\left(\mathrm{GL}_{n}\right) & \cong\left(\left(\mathrm{SL}_{n} \times \mathbb{C}^{*}\right) / / \mathbb{Z}_{n}\right)^{r} / / \mathrm{SL}_{n} \\
& \cong\left(\left(\mathrm{SL}_{n}^{r} \times\left(\mathbb{C}^{*}\right)^{r}\right) / / \mathbb{Z}_{n}^{r}\right) / / \mathrm{SL}_{n} \\
& \cong\left(\left(\mathrm{SL}_{n}^{r} \times\left(\mathbb{C}^{*}\right)^{r}\right) / / \mathrm{SL}_{n}\right) / / \mathbb{Z}_{n}^{r} \cong \mathfrak{X}_{r}\left(\mathrm{SL}_{n}\right) \times \mathbb{Z}_{n}^{r}\left(\mathbb{C}^{*}\right)^{r},
\end{aligned}
$$

since the action of $\mathbb{Z}_{n}^{r}$ commutes with the action of $\mathrm{SL}_{n}$ which is trivial on $\left(\mathbb{C}^{*}\right)^{r}$.

In the same way we obtain the other "twisted product" isomorphism $\mathfrak{X}_{r}\left(\mathrm{U}_{n}\right) \cong$ $\mathfrak{X}_{r}\left(\mathrm{SU}_{n}\right) \times_{\mathbb{Z}_{n}^{r}}\left(S^{1}\right)^{r}$.

This result provides an explicit way to write $\mathfrak{X}_{r}\left(\mathrm{GL}_{n}\right)$ as a $\mathfrak{X}_{r}\left(\mathrm{SL}_{n}\right)$-bundle over the algebraic $r$-torus $\left(\mathbb{C}^{*}\right)^{r}$ and $\mathfrak{X}_{r}\left(\mathrm{U}_{n}\right)$ as a $\mathfrak{X}_{r}\left(\mathrm{SU}_{n}\right)$-bundle over the geometric $r$-torus $\left(S^{1}\right)^{r}$.

There are a number of consequences to Theorem 2.4.

Corollary 2.5. $\mathfrak{X}_{r}\left(\mathrm{U}_{n}\right)$, respectively $\mathfrak{X}_{r}\left(\mathrm{GL}_{n}\right)$, is a manifold whenever $\mathfrak{X}_{r}\left(\mathrm{SU}_{n}\right)$, respectively $\mathfrak{X}_{r}\left(\mathrm{SL}_{n}\right)$, is a manifold.

Proof. The action of $\mathbb{Z}_{n}^{r}$ is free and proper.

Corollary 2.6. $\mathfrak{X}_{r}\left(\mathrm{GL}_{n}\right)$ and $\mathfrak{X}_{r}\left(\mathrm{SL}_{n}\right) \times\left(\mathbb{C}^{*}\right)^{r}$ are étale equivalent.

Proof. First note that $\mathrm{SL}_{n}^{r} \times\left(\mathbb{C}^{*}\right)^{r}$ is smooth and hence a normal variety. This implies (see [Drézet 2004]) that $\left(\mathrm{SL}_{n}^{r} \times\left(\mathbb{C}^{*}\right)^{r}\right) / / \mathrm{SL}_{n}=\mathfrak{X}_{r}\left(\mathrm{SL}_{n}\right) \times\left(\mathbb{C}^{*}\right)^{r}$ is also normal. However, the GIT projection

$$
\mathfrak{X}_{r}\left(\mathrm{SL}_{n}\right) \times\left(\mathbb{C}^{*}\right)^{r} \rightarrow \mathfrak{X}_{r}\left(\mathrm{SL}_{n}\right) \times \mathbb{Z}_{n}^{r}\left(\mathbb{C}^{*}\right)^{r}
$$

is then étale because $\mathbb{Z}_{n}^{r}$ is finite and acts freely [ibid.]. Then by Theorem 2.4 $\mathfrak{X}_{r}\left(\mathrm{GL}_{n}\right) \cong \mathfrak{X}_{r}\left(\mathrm{SL}_{n}\right) \times_{\mathbb{Z}_{n}^{r}}\left(\mathbb{C}^{*}\right)^{r}$ which establishes the result.

Corollary 2.7. Let $[\rho] \in \mathfrak{X}_{r}\left(\mathrm{SL}_{n}\right)$ and let $[\psi] \in \mathfrak{X}_{r}\left(\mathrm{SU}_{n}\right)$. Then:

(i) $[\rho] \in \mathfrak{X}_{r}\left(\mathrm{SL}_{n}\right)^{\text {sing }}$ if and only if $[\rho] \in \mathfrak{X}_{r}\left(\mathrm{GL}_{n}\right)^{\text {sing }}$.

(ii) $[\rho] \in \mathfrak{X}_{r}\left(\mathrm{SL}_{n}\right)^{\mathrm{sm}}$ if and only if $[\rho] \in \mathfrak{X}_{r}\left(\mathrm{GL}_{n}\right)^{\mathrm{sm}}$.

(iii) $[\psi] \in \mathfrak{X}_{r}\left(\mathrm{SU}_{n}\right)^{\text {sing }}$ if and only if $[\psi] \in \mathfrak{X}_{r}\left(\mathrm{U}_{n}\right)^{\text {sing }}$.

(iv) $[\psi] \in \mathfrak{X}_{r}\left(\mathrm{SU}_{n}\right)^{\mathrm{sm}}$ if and only if $[\psi] \in \mathfrak{X}_{r}\left(\mathrm{U}_{n}\right)^{\mathrm{sm}}$. 
Proof. First let $[\rho] \in \mathfrak{X}_{r}\left(\mathrm{SL}_{n}\right)$. Corollary 2.6 tells that $\mathfrak{X}_{r}\left(\mathrm{SL}_{n}\right) \times\left(\mathbb{C}^{*}\right)^{r} \rightarrow \mathfrak{X}_{r}\left(\mathrm{GL}_{n}\right)$ is an étale equivalence and such mappings preserve tangent spaces, we conclude

$$
T_{[\rho]}\left(\mathfrak{X}_{r}\left(\mathrm{GL}_{n}\right)\right) \cong T_{[\rho]}\left(\mathfrak{X}_{r}\left(\mathrm{SL}_{n}\right) \times\left(\mathbb{C}^{*}\right)^{r}\right) \cong T_{[\rho]}\left(\mathfrak{X}_{r}\left(\mathrm{SL}_{n}\right)\right) \oplus \mathbb{C}^{r} .
$$

By counting dimensions and noticing that

$$
\operatorname{dim}_{\mathbb{C}}\left(\mathfrak{X}_{r}\left(\mathrm{GL}_{n}\right)\right)=\operatorname{dim}_{\mathbb{C}}\left(\mathfrak{X}_{r}\left(\mathrm{SL}_{n}\right)\right)+r,
$$

results (i) and (ii) follow.

Results (iii) and (iv) follow from (i) and (ii) and the additional observations that $\operatorname{dim}_{\mathbb{C}}\left(\mathfrak{X}_{r}\left(K_{\mathbb{C}}\right)\right)=\operatorname{dim}_{\mathbb{R}}\left(\mathfrak{X}_{r}(K)\right)$ and $\operatorname{dim}_{\mathbb{C}}\left(T_{[\psi]}\left(\mathfrak{X}_{r}\left(K_{\mathbb{C}}\right)\right)\right)=\operatorname{dim}_{\mathbb{R}}\left(T_{[\psi]}\left(\mathfrak{X}_{r}(K)\right)\right)$.

Corollary 2.8. We have the following isomorphisms of character varieties:

(i) $\mathfrak{X}_{r}\left(\mathrm{PSL}_{n}\right) \cong \mathfrak{X}_{r}\left(\mathrm{SL}_{n}\right) / / \mathbb{Z}_{n}^{r}$ in the category of algebraic varieties.

(ii) $\mathfrak{X}_{r}\left(\mathrm{PU}_{n}\right) \cong \mathfrak{X}_{r}\left(\mathrm{SU}_{n}\right) / \mathbb{Z}_{n}^{r}$ in the category of semialgebraic sets.

Proof. From the previous theorem we have

$$
\mathfrak{X}_{r}\left(\mathrm{GL}_{n}\right) \cong \mathfrak{X}_{r}\left(\mathrm{SL}_{n}\right) \times \mathbb{Z}_{n}^{r}\left(\mathbb{C}^{*}\right)^{r} .
$$

Taking the quotient of both sides by $\left(\mathbb{C}^{*}\right)^{r}$ we can conclude $\mathfrak{X}_{r}\left(\mathrm{PSL}_{n}\right) \cong \mathfrak{X}_{r}\left(\mathrm{SL}_{n}\right) / / \mathbb{Z}_{n}^{r}$. More precisely letting $\mu=\left(\mu_{1}, \ldots, \mu_{r}\right) \in\left(\mathbb{C}^{*}\right)^{r}$ act only on the second factor of $\mathfrak{X}_{r}\left(\mathrm{SL}_{n}\right) \times\left(\mathbb{C}^{*}\right)^{r}$,

$$
\mu \cdot\left(\left[\left(A_{1}, \ldots, A_{r}\right)\right],\left(\lambda_{1}, \ldots, \lambda_{r}\right)\right)=\left(\left[\left(A_{1}, \ldots, A_{r}\right)\right],\left(\mu_{1} \lambda_{1}, \ldots, \mu_{r} \lambda_{r}\right)\right),
$$

and going through the isomorphisms in (3), one gets that the action on $\mathfrak{X}_{r}\left(\mathrm{GL}_{n}\right)$ corresponds to scalar multiplication of each entry, so we obtain

$$
\begin{aligned}
\mathfrak{X}_{r}\left(\mathrm{PSL}_{n}\right) \cong \mathfrak{X}_{r}\left(\mathrm{GL}_{n}\right) / /\left(\mathbb{C}^{*}\right)^{r} & \cong\left(\mathfrak{X}_{r}\left(\mathrm{SL}_{n}\right) \times \mathbb{Z}_{n}^{r}\left(\mathbb{C}^{*}\right)^{r}\right) / /\left(\mathbb{C}^{*}\right)^{r} \\
& \cong\left(\left(\mathfrak{X}_{r}\left(\mathrm{SL}_{n}\right) \times\left(\mathbb{C}^{*}\right)^{r}\right) / / \mathbb{Z}_{n}^{r}\right) / /\left(\mathbb{C}^{*}\right)^{r} \\
& \cong\left(\left(\mathfrak{X}_{r}\left(\mathrm{SL}_{n}\right) \times\left(\mathbb{C}^{*}\right)^{r}\right) / /\left(\mathbb{C}^{*}\right)^{r}\right) / / \mathbb{Z}_{n}^{r} \cong \mathfrak{X}_{r}\left(\mathrm{SL}_{n}\right) / / \mathbb{Z}_{n}^{r},
\end{aligned}
$$

as wanted. The other statement is analogous.

2B. Examples. We use the results in Section $2 \mathrm{~A}$ and the theorems from [Florentino and Lawton 2009] to describe the homeomorphism types of the examples of $G$ character varieties of $\mathrm{F}_{r}$ known to be manifolds with boundary. Let $\bar{B}_{n}$ denote a closed real ball of indicated dimension, and let $\{*\}$ denote the space consisting of one point.

One can show that whenever $\phi: \mathfrak{X}_{r}\left(\mathrm{SL}_{n}\right) \rightarrow M$ is an isomorphism (as affine varieties), then $\mathfrak{X}_{r}\left(\mathrm{SU}_{n}\right) \cong \phi\left(\mathfrak{X}_{r}\left(\mathrm{SU}_{n}\right)\right) \subset M$ (as semialgebraic sets) by restricting $\phi$ to $\mathfrak{X}_{r}\left(\mathrm{SU}_{n}\right) \subset \mathfrak{X}_{r}\left(\mathrm{SL}_{n}\right)$ [Procesi and Schwarz 1985].

We first consider the trivial case $(r, n)=(r, 1)$. In this case the conjugation action 
is trivial, and thus we deduce the following table of moduli of $(r, 1)$-representations:

\begin{tabular}{|c|c|c|}
\hline & fixed determinant & nonfixed determinant \\
\hline complex & $\mathfrak{X}_{r}\left(\mathrm{SL}_{1}\right) \cong\{*\}$ & $\mathfrak{X}_{r}\left(\mathrm{GL}_{1}\right) \cong\left(\mathbb{C}^{*}\right)^{r}$ \\
compact & $\mathfrak{X}_{r}\left(\mathrm{SU}_{1}\right) \cong\{*\}$ & $\mathfrak{X}_{r}\left(\mathrm{U}_{1}\right) \cong\left(S^{1}\right)^{r}$ \\
\hline
\end{tabular}

We next consider the case $r=1$. The coefficients of the characteristic polynomial of a matrix $X,\left\{c_{1}(X), \ldots, c_{n-1}(X), \operatorname{det}(X)\right\}$, define conjugate invariant regular mappings $\mathfrak{X}_{1}\left(\mathrm{SL}_{n}\right) \rightarrow \mathbb{C}^{n-1}$ and $\mathfrak{X}_{1}\left(\mathrm{GL}_{n}\right) \rightarrow \mathbb{C}^{n-1} \times \mathbb{C}^{*}$ which are isomorphisms. Thus we get this table of moduli of $(1, n)$-representations:

\begin{tabular}{|l|c|c|}
\hline & fixed determinant & nonfixed determinant \\
\hline complex & $\mathfrak{X}_{1}\left(\mathrm{SL}_{n}\right) \cong \mathbb{C}^{n-1}$ & $\mathfrak{X}_{1}\left(\mathrm{GL}_{n}\right) \cong \mathbb{C}^{n-1} \times \mathbb{C}^{*}$ \\
compact & $\mathfrak{X}_{1}\left(\mathrm{SU}_{n}\right) \cong \bar{B}_{n-1}$ & $\mathfrak{X}_{1}\left(\mathrm{U}_{n}\right) \cong \bar{B}_{n-1} \times S^{1}$ \\
\hline
\end{tabular}

Remark 2.9. With respect to the second table, each of the four families of moduli spaces contains no irreducible representations, yet each space is smooth. For this reason these moduli spaces should perhaps be regarded as everywhere singular, since we will see that irreducibles will generally be smooth points for $r \geq 2$.

In the $r=2$ case we have a well-known isomorphism $\mathfrak{X}_{2}\left(\mathrm{SL}_{2}\right) \rightarrow \mathbb{C}^{3}$ given by $[(A, B)] \mapsto(\operatorname{tr}(A), \operatorname{tr}(B), \operatorname{tr}(A B))$; see [Goldman 2009; Vogt 1889; Fricke and Klein 1912]. More generally there is an isomorphism $\mathfrak{g l}(2, \mathbb{C})^{2} / / \mathrm{PGL}_{2} \rightarrow \mathbb{C}^{5}$ given by

$$
[(A, B)] \mapsto(\operatorname{tr}(A), \operatorname{tr}(B), \operatorname{tr}(A B), \operatorname{det}(A), \operatorname{det}(B)) .
$$

Therefore we get the following moduli of $(2,2)$-representations:

\begin{tabular}{|c|c|c|}
\hline & fixed determinant & nonfixed determinant \\
\hline complex & $\mathfrak{X}_{2}\left(\mathrm{SL}_{2}\right) \cong \mathbb{C}^{3}$ & $\mathfrak{X}_{2}\left(\mathrm{GL}_{2}\right) \cong \mathbb{C}^{3} \times\left(\mathbb{C}^{*} \times \mathbb{C}^{*}\right)$ \\
compact & $\mathfrak{X}_{2}\left(\mathrm{SU}_{2}\right) \cong \bar{B}_{3}$ & $\mathfrak{X}_{2}\left(\mathrm{U}_{2}\right) \cong \bar{B}_{3} \times\left(S^{1} \times S^{1}\right)$ \\
\hline
\end{tabular}

In [Florentino and Lawton 2009] the following fixed determinant cases are established:

\begin{tabular}{|l|c|c|}
\hline & fixed determinant & nonfixed determinant \\
\hline compact $(3,2)$ & $\mathfrak{X}_{3}\left(\mathrm{SU}_{2}\right) \cong S^{6}$ & $\mathfrak{X}_{3}\left(\mathrm{U}_{2}\right) \cong S^{6} \times_{\mathbb{Z}_{2}^{3}}\left(S^{1} \times S^{1} \times S^{1}\right)$ \\
compact $(2,3)$ & $\mathfrak{X}_{2}\left(\mathrm{SU}_{3}\right) \cong S^{8}$ & $\mathfrak{X}_{2}\left(\mathrm{U}_{3}\right) \cong S^{8} \times_{\mathbb{Z}_{3}^{2}}\left(S^{1} \times S^{1}\right)$ \\
\hline
\end{tabular}

Remark 2.10. The complex $(3,2)$ and $(2,3)$ cases are left out in this last table since we will show they are not manifolds. In each of these cases, the complex 
moduli space of fixed determinant is a branched double cover of complex affine space which deformation retract to a sphere. The explicit scheme structures are known as well. See [Florentino and Lawton 2009; Lawton 2007].

We conjectured in [Florentino and Lawton 2009] that this covers all the cases where a topological manifold with boundary can arise. We will prove this conjecture in Section 4.

\section{Singularities}

3A. Algebro-geometric singularities. There are a number of equivalent ways to describe smoothness for affine varieties.

Let $X=V\left(f_{1}, \ldots, f_{k}\right) \subset \mathbb{C}^{n}$ be an affine variety. Then its tangent space at the point $\boldsymbol{p}=\left(p_{1}, \ldots, p_{n}\right) \in X$ is the vector space

$$
T_{\boldsymbol{p}}(X)=\left\{\left(v_{1}, \ldots, v_{n}\right) \in \mathbb{C}^{n}\left|\sum_{j=1}^{n} \frac{\partial f_{i}}{\partial x_{j}}\right|_{\boldsymbol{p}}\left(v_{j}-p_{j}\right)=0 \text { for all } i\right\} .
$$

This coincides with the more general definition $T_{p}(X)=\left(\mathfrak{m}_{p} / \mathfrak{m}_{p}^{2}\right)^{*}$, which is the dual to the cotangent space $\mathfrak{m}_{p} / \mathfrak{m}_{p}^{2}$, where $\mathfrak{m}_{p}$ is a maximal ideal in $\mathbb{C}[X]$ corresponding to $\boldsymbol{p}$ by Hilbert's Nullstellensatz.

Definition 3.1. The singular locus of $X$ is defined to be

$$
X^{\text {sing }}=\left\{\boldsymbol{p} \in X \mid \operatorname{dim}_{\mathbb{C}} T_{\boldsymbol{p}}(X)>\operatorname{dim}_{\mathrm{Krull}} X\right\} .
$$

The complement of this set, $X-X^{\text {sing }}$, is a complex manifold. If $X$ is irreducible, then $X$ is path-connected and furthermore $X-X^{\text {sing }}$ is likewise path-connected. See [Shafarevich 1994].

Let $c=n-\operatorname{dim}_{\mathrm{Krull}} X$. And let $J$ be the $k \times n$ Jacobian matrix of partial derivatives of the $k$ relations defining $X \subset \mathbb{C}^{n}$. We can assume $n$ is minimal. Then $X^{\text {sing }}$ is concretely realized as the affine variety determined by the determinant of the $c \times c$ minors of $J$. This ideal is referred to as the Jacobian ideal. In this way, $X^{\text {sing }}$ is seen to be a proper subvariety of $X$.

For example, in [Heusener and Porti 2004] it is shown (for $r \geq 3$ ) that

$$
\mathfrak{X}_{r}\left(\mathrm{SL}_{2}\right)^{\text {sing }}=\mathfrak{X}_{r}\left(\mathrm{SL}_{2}\right)^{\text {red }} .
$$

In [Lawton 2007], explicitly computing the Jacobian ideal, a similar result is also shown: $\mathfrak{X}_{2}\left(\mathrm{SL}_{3}\right)^{\text {red }}=\mathfrak{X}_{2}\left(\mathrm{SL}_{3}\right)^{\text {sing }}$.

3B. Tangent spaces. Let $\mathfrak{g}$ be the Lie algebra of $G$. Having addressed the $r=1$ and $n=1$ cases, we now assume that $r, n \geq 2$.

The following two lemmas are classical, and in fact are true for any algebraic Lie group over $\mathbb{R}$ or $\mathbb{C}$. See [Weil 1964]. For a representation $\rho: \mathrm{F}_{r} \rightarrow G$, let us 
denote by $\mathfrak{g}_{\mathrm{Ad}_{\rho}}$ the $\mathrm{F}_{r}$-module $\mathfrak{g}$ with the adjoint action via $\rho$. That is, any word $w \in \mathrm{F}_{r}$ acts as $w \cdot X=\operatorname{Ad}_{\rho(w)} X=\rho(w) X \rho(w)^{-1}$, for $X \in \mathfrak{g}$. Consider the cocycles, coboundaries and cohomology of $\mathrm{F}_{r}$ with coefficients in this module. Explicitly:

$$
\begin{aligned}
Z^{1}\left(\mathrm{~F}_{r} ; \mathfrak{g}_{\mathrm{Ad}_{\rho}}\right) & :=\left\{u: \mathrm{F}_{r} \rightarrow \mathfrak{g} \mid u(x y)=u(x)+\operatorname{Ad}_{\rho(x)} u(y)\right\}, \\
B^{1}\left(\mathrm{~F}_{r} ; \mathfrak{g}_{\mathrm{Ad}_{\rho}}\right) & :=\left\{u: \mathrm{F}_{r} \rightarrow \mathfrak{g} \mid u(x)=\operatorname{Ad}_{\rho(x)} X-X \text { for some } X \in \mathfrak{g}\right\}, \\
H^{1}\left(\mathrm{~F}_{r} ; \mathfrak{g}_{\mathrm{Ad}_{\rho}}\right) & :=Z^{1}\left(\mathrm{~F}_{r} ; \mathfrak{g}_{\mathrm{Ad}_{\rho}}\right) / B^{1}\left(\mathrm{~F}_{r} ; \mathfrak{g}_{\mathrm{Ad}_{\rho}}\right) .
\end{aligned}
$$

Lemma 3.2. Let $G$ be any algebraic Lie group over $\mathbb{R}$ or $\mathbb{C}$.

$$
T_{\rho}\left(\Re_{r}(G)\right) \cong \mathfrak{g}^{r} \cong Z^{1}\left(\mathrm{~F}_{r} ; \mathfrak{g}_{\mathrm{Ad}_{\rho}}\right) .
$$

Let $\operatorname{Orb}_{\rho}=\left\{g \rho g^{-1} \mid g \in G\right\}$ be the $G$-orbit of $\rho$, and let $\operatorname{Stab}_{\rho}=\left\{g \in G \mid g \rho g^{-1}=\right.$ $\rho$ \} be the $G$-stabilizer (or isotropy subgroup).

Lemma 3.3. Let $G$ be any algebraic Lie group over $\mathbb{R}$ or $\mathbb{C}$.

$$
T_{\rho}\left(\mathrm{Orb}_{\rho}\right) \cong \mathfrak{g} /\left\{X \in \mathfrak{g} \mid \operatorname{Ad}_{\rho(x)} X=X\right\} \cong B^{1}\left(\mathrm{~F}_{r} ; \mathfrak{g}_{\operatorname{Ad}_{\rho}}\right) .
$$

It is not always the case that the tangent space to the quotient is the quotient of tangent spaces. Consider representations from the free group of rank 1 into $\mathrm{SL}_{3}$. The ring of invariants is two dimensional and the ring is generated by $\operatorname{tr}(X)$ and $\operatorname{tr}\left(X^{-1}\right)$. So the ideal is zero and the ring is free. Consequently it is smooth and the representation sending everything to the identity (having maximal stabilizer) is a nonsingular point. This illustrates that there can be smooth points in the quotient that have positive-dimensional stabilizer. At these points, $T_{\rho}\left(\mathfrak{R}_{r}(G) / / G\right) \not$ $T_{\rho}\left(\mathfrak{R}_{r}(G)\right) / T_{\rho}\left(\mathrm{Orb}_{\rho}\right)$, seen by simply comparing dimensions.

We also note that if we replace free groups by finitely generated groups $\Gamma$ then the above isomorphisms require a more careful treatment due to the possible existence of nilpotents in the coordinate ring of the scheme associated to $\mathfrak{R}_{\Gamma}(G)$ [Sikora 2012].

Recall that $\mathfrak{R}_{r}(G)^{s}$ is the set of irreducible representations, and $\mathfrak{X}_{r}(G)^{s}=$ $\mathfrak{R}_{r}(G)^{s} / G$. An action is called locally free if the stabilizer is finite, and is called proper if the action $G \times X \rightarrow X \times X$ is a proper mapping. In general, the quotient by a proper locally free action of a reductive group on a smooth manifold is an orbifold (a space locally modeled on finite quotients of $\mathbb{R}^{n}$ ).

The following lemma can be found in [Johnson and Millson 1987, pp. 54-57]. See also [Goldman 1990; 1984].

Lemma 3.4. Let $G$ be reductive and $r, n \geq 2$. The $\mathrm{P} G$ action on $\mathfrak{R}_{r}(G)^{s}$ is locally free and proper.

Therefore, $\mathfrak{R}_{r}(G)^{s} / G=\mathfrak{R}_{r}(G)^{s} / \mathrm{P} G$ are orbifolds. 
Lemma 3.5. For $G$ equal to $\mathrm{SL}_{n}, \mathrm{GL}_{n}, \mathrm{SU}_{n}$, or $\mathrm{U}_{n}$ and $r, n \geq 2$, the associated $\mathrm{PG}$ action on $\mathfrak{R}_{r}(G)^{s}$ is free. Therefore, in these cases $\mathfrak{R}_{r}(G)^{s} / G$ is a smooth manifold.

Proof. Let $\rho=\left(X_{1}, \ldots, X_{r}\right) \in \mathfrak{R}_{r}(G)^{s}$. Then by Burnside's theorem [Lang 2002] the collection $\left\{X_{1}, \ldots, X_{r}\right\}$ generates all of $n \times n$ matrices $M_{n \times n}$ as an algebra, since $r>1$ and they form an irreducible set of matrices. Suppose there exists $g \in G$ so that for all $1 \leq k \leq r$ we have $g X_{k} g^{-1}=X_{k}$. Then $g$ stabilizes all of $M_{n \times n}$.

Consider $M=\mathbb{C}^{n}$ as a module over $R=M_{n \times n}$. Clearly, $M$ is a simple module since no nontrivial proper subspaces are left invariant by all matrices. Let $f_{g}$ be the automorphism of $\mathbb{C}^{n}$ defined by mapping $v \mapsto g v$. Then $f_{g}$ defines an $R$-module automorphism of $M$ since $g$ stabilizes all of $R$. Thus by Schur's lemma the action of $g$ is equal to the action of a scalar; that is, $g$ is central.

Lemma 3.5 and Lemma 3.16 (see Section 3D) together immediately imply the following corollary.

Corollary 3.6. Let $G=\mathrm{SL}_{n}, \mathrm{GL}_{n}, \mathrm{SU}_{n}$, or $\mathrm{U}_{n}$. If $[\rho] \in \mathfrak{X}_{r}(G)^{s}$ and $r, n \geq 2$, then

$$
T_{[\rho]}\left(\mathfrak{X}_{r}(G)\right) \cong H^{1}\left(\mathrm{~F}_{r} ; \mathfrak{g}_{\mathrm{Ad}_{\rho}}\right) .
$$

For $G=\mathrm{SL}_{n}$ we can calculate that $\operatorname{dim}_{\mathbb{C}} \mathfrak{X}_{r}(G)^{s}=\left(n^{2}-1\right)(r-1)$ and for $K=\mathrm{SU}_{n}$, we have $\operatorname{dim}_{\mathbb{R}} \mathfrak{X}_{r}(K)^{s}=\left(n^{2}-1\right)(r-1)$. Likewise, for $G=\mathrm{GL}_{n}$ we calculate $\operatorname{dim}_{\mathbb{C}} \mathfrak{X}_{r}(G)^{s}=n^{2}(r-1)+1$ and for $K=\mathrm{U}_{n}, \operatorname{dim}_{\mathbb{R}} \mathfrak{X}_{r}(K)^{s}=n^{2}(r-1)+1$.

Let $\mathfrak{X}_{r}(G)^{\mathrm{sm}}=\mathfrak{X}_{r}(G)-\mathfrak{X}_{r}(G)^{\text {sing }}$ be the smooth stratum, which is a complex manifold, open and dense as a subspace of $\mathfrak{X}_{r}(G)$. The calculation of dimensions above and Corollary 3.6 imply the following lemma which expresses the fact that the irreducibles not only form a smooth manifold but are naturally contained in the smooth stratum of the variety.

Lemma 3.7. Let $r, n \geq 2$ and $G$ be one of $\mathrm{SL}_{n}$ or $\mathrm{GL}_{n}$. Then the following equivalent statements hold:

(i) $\mathfrak{X}_{r}(G)^{s} \subset \mathfrak{X}_{r}(G)^{\mathrm{sm}}$.

(ii) $\mathfrak{X}_{r}(G)^{\text {sing }} \subset \mathfrak{X}_{r}(G)^{\text {red }}$.

The next lemmas address important technical points that we will need in our proofs.

Lemma 3.8. $\mathfrak{X}_{r}(G)^{\text {red }}$ is an algebraic set; that is, a subvariety of $\mathfrak{X}_{r}(G)$.

Proof. The irreducibles are exactly the GIT stable points (zero dimensional stabilizer and closed orbits) and in general these are Zariski open, which implies the complement is an algebraic set [Dolgachev 2003].

Lemma 3.9. Suppose there exists a set $\mathbb{O} \subset \mathfrak{X}_{r}(G)^{\text {sing }} \cap \mathfrak{X}_{r}(G)^{\text {red }}$ that is dense with respect to the ball topology in $\mathfrak{X}_{r}(G)^{\mathrm{red}}$. Then $\mathfrak{X}_{r}(G)^{\operatorname{sing}}=\mathfrak{X}_{r}(G)^{\mathrm{red}}$. 
Proof. Since both $\mathfrak{X}_{r}(G)^{\text {sing }} \subset \mathfrak{X}_{r}(G)^{\text {red }}$ are subvarieties (by Lemmas 3.7 and 3.8), $\mathcal{O}$ is dense in both with respect to the ball topology. This follows since $\mathbb{O}$ is dense in $\mathfrak{X}_{r}(G)^{\text {red }}$ with respect to the ball topology and $\mathcal{C} \subset \mathfrak{X}_{r}(G)^{\text {sing }} \cap \mathfrak{X}_{r}(G)^{\text {red }}$. Thus $\mathfrak{X}_{r}(G)^{\text {sing }}=\overline{\mathbb{O}}=\mathfrak{X}_{r}(G)^{\text {red }}$, where $\overline{0}$ is the closure of $\mathbb{O}$ in $\mathfrak{X}_{r}(G)$ with respect to the (metric) ball topology.

A set as in Lemma 3.9 was called an adherence set in [Heusener and Porti 2004].

3C. Denseness of reducibles with minimal stabilizer. Now consider the following subvarieties of reducibles. Recall that the 0 vector space is not considered to be an irreducible subrepresentation.

Definition 3.10. Define $U_{r, n} \subset \mathfrak{X}_{r}\left(\mathrm{GL}_{n}\right)^{\text {red }}$ and $W_{r, n} \subset \mathfrak{X}_{r}\left(\mathrm{SL}_{n}\right)^{\text {red }}$ by:

$$
\begin{aligned}
U_{r, n} & =\left\{\left[\rho_{1} \oplus \rho_{2}\right] \in \mathfrak{X}_{r}\left(\mathrm{GL}_{n}\right): \rho_{i}: \mathrm{F}_{r} \rightarrow \mathrm{GL}_{n_{i}} \text { are irreducible }\right\} \\
W_{r, n} & =\left\{\left[\rho_{1} \oplus \rho_{2}\right] \in \mathfrak{X}_{r}\left(\mathrm{SL}_{n}\right): \rho_{i}: \mathrm{F}_{r} \rightarrow \mathrm{GL}_{n_{i}} \text { are irreducible }\right\},
\end{aligned}
$$

where we consider all possible decompositions $n=n_{1}+n_{2}$, with $n_{i}>0$.

Note that a given $\rho \in U_{r, n}$ uniquely determines the integers $n_{1}$ and $n_{2}$, up to permutation. We will refer to this situation by saying that $\rho$ is of reduced type $\left[n_{1}, n_{2}\right]$. Similar remarks and terminology apply to $W_{r, n}$.

It is clear that

$$
\mathfrak{X}_{r}\left(\mathrm{SL}_{n}\right)^{\mathrm{red}}=\mathfrak{X}_{r}\left(\mathrm{GL}_{n}\right)^{\mathrm{red}} \cap \mathfrak{X}_{r}\left(\mathrm{SL}_{n}\right)
$$

and that

$$
W_{r, n}=U_{r, n} \cap \mathfrak{X}_{r}\left(\mathrm{SL}_{n}\right) .
$$

The following lemma is likewise clear by the proof of Lemma 3.5.

Lemma 3.11. A representation $\rho$ is in $U_{r, n}$ if and only if $\operatorname{Stab}_{\rho} \cong\left(\mathbb{C}^{*}\right)^{2}$. Also, $\rho \in W_{r, n}$ if and only if $\operatorname{Stab}_{\rho} \cong \mathbb{C}^{*}$.

The strategy is now to show that $U_{r, n}$ and $W_{r, n}$ contain only singularities. However, we must first establish the following lemma.

Lemma 3.12. Let $r, n \geq 2$. $U_{r, n}$ is dense in $\mathfrak{X}_{r}\left(\mathrm{GL}_{n}\right)^{\text {red }}$ with respect to the ball topology.

Proof. When $n=2, U_{r, n}$ coincides with $\mathfrak{X}_{r}\left(\mathrm{GL}_{n}\right)^{\text {red }}$, since any completely reducible representation is of reduced type $[1,1]$. So we assume $n \geq 3$. Let $\rho \in[\rho] \in$ $\mathfrak{X}_{r}\left(\mathrm{GL}_{n}\right)^{\text {red }}$ have at least three irreducible blocks; that is, $\rho=\rho_{1} \oplus \rho_{2} \oplus \rho_{3}$ where $\rho_{1}$ and $\rho_{2}$ are irreducible and $\rho_{3}$ is semisimple. In other words, $[\rho] \in \mathfrak{X}_{r}\left(\mathrm{GL}_{n}\right)^{\text {red }}-U_{r, n}$.

Then $\rho_{2} \oplus \rho_{3}$ is a semisimple representation into $\mathrm{GL}_{k}$ for some $k$. Since the irreducible representations $\mathrm{F}_{r} \rightarrow \mathrm{GL}_{k}$ are dense (here we use $r>1$ ), there exists an 
irreducible sequence $\sigma_{j} \in \operatorname{Hom}\left(\mathrm{F}_{r}, \mathrm{GL}_{k}\right)$ satisfying

$$
\lim _{j \rightarrow \infty} \sigma_{j}=\rho_{2} \oplus \rho_{3},
$$

which in turn implies

$$
\lim _{j \rightarrow \infty} \rho_{1} \oplus \sigma_{j}=\rho_{1} \oplus \rho_{2} \oplus \rho_{3}=\rho,
$$

where $\rho_{1} \oplus \sigma_{j}$ is in $U_{r, n}$. Thus we have a sequence $\left[\rho_{1} \oplus \sigma_{j}\right] \in U_{r, n} \subset \mathfrak{X}_{r}\left(\mathrm{GL}_{n}\right)^{\text {red }}$ whose limit is $\left[\rho_{1} \oplus \rho_{2} \oplus \rho_{3}\right]$. This shows that $U_{r, n}$ is dense in $\mathfrak{X}_{r}\left(\mathrm{GL}_{n}\right)^{\text {red }}$ and proves the lemma.

Corollary 3.13. Let $r, n \geq 2$. Then $W_{r, n}$ is dense in $\mathfrak{X}_{r}\left(\mathrm{SL}_{n}\right)^{\text {red }}$ with respect to the ball topology.

Proof. First we show that $\mathfrak{X}_{r}\left(\mathrm{SL}_{n}\right)^{\text {red }} \subset \overline{W_{r, n}}$. Using the previous lemma and Equation (4), let

$$
\begin{aligned}
{[\rho] \in \mathfrak{X}_{r}\left(\mathrm{SL}_{n}\right)^{\mathrm{red}} } & =\mathfrak{X}_{r}\left(\mathrm{SL}_{n}\right) \cap \mathfrak{X}_{r}\left(\mathrm{GL}_{n}\right)^{\mathrm{red}} \\
& =\mathfrak{X}_{r}\left(\mathrm{SL}_{n}\right) \cap \overline{U_{r, n}} .
\end{aligned}
$$

Then, we can write $\rho=\lim \sigma_{j}$, where $\sigma_{j}=\rho_{1}^{(j)} \oplus \rho_{2}^{(j)} \in U_{r, n}$ is of reduced type $\left[n_{1}, n_{2}\right]$. Let us write $\lambda_{j}:=\operatorname{det} \rho_{1}^{(j)} \operatorname{det} \rho_{2}^{(j)}$. Since the limit is a well-defined point $[\rho] \in \mathfrak{X}_{r}\left(\mathrm{SL}_{n}\right)^{\text {red }}$, we can arrange for the sequence to be in $W_{r, n}$ as follows. Letting $\alpha_{j}=\left(1 / \lambda_{j}\right)^{1 / n_{1}}$ (for any choice of branch cut), we can also write $\rho=\lim \eta_{j}$ where $\eta_{j}=\left(\rho_{1}^{(j)} \alpha_{j}\right) \oplus \rho_{2}^{(j)} \in W_{r, n}$, (since now $\eta_{j}$ has unit determinant), from which one sees that $\rho \in \overline{W_{r, n}}$, as wanted. Finally, we get:

$$
\begin{aligned}
\mathfrak{X}_{r}\left(\mathrm{SL}_{n}\right)^{\mathrm{red}} & \subset \overline{W_{r, n}}=\overline{\mathfrak{X}_{r}\left(\mathrm{SL}_{n}\right) \cap U_{r, n}} \\
& \subset \mathfrak{X}_{r}\left(\mathrm{SL}_{n}\right) \cap \overline{U_{r, n}} \\
& =\mathfrak{X}_{r}\left(\mathrm{SL}_{n}\right)^{\mathrm{red}},
\end{aligned}
$$

which implies all these sets coincide, finishing the proof. Here, we used the standard fact that the closure of an intersection is contained in the intersection of the closures, and that $\mathfrak{X}_{r}\left(\mathrm{SL}_{n}\right)$ is closed in $\mathfrak{X}_{r}\left(\mathrm{GL}_{n}\right)$.

3D. The Luna slice theorem and the Zariski tangent space. We now prove a strong lemma, first proved in [Heusener and Porti 2004] and later in more generality in [Sikora 2012], which tells exactly how to understand the Zariski tangent space at a general free group representation. For a similar result see also [Drézet 2004, p. 45]. To that end, we review the Luna slice theorem [1973]. We recommend [Drézet 2004] for a good exposition.

Following [Schwarz 2004], we define an étale map between complex affine varieties as a local analytic isomorphism in the ball topology. 
Theorem 3.14 (weak Luna slice theorem at smooth points). Let $G$ be a reductive algebraic group acting on an affine variety $X$. Let $x \in X$ be a smooth point with $\mathrm{Orb}_{x}$ closed. Then there exists a subvariety $x \in V \subset X$, and $\mathrm{Stab}_{x}$-invariant étale morphism $\phi: V \rightarrow T_{x} V$ satisfying:

(i) $V$ is locally closed, affine, smooth, and $\mathrm{Stab}_{x}$-stable.

(ii) $V \hookrightarrow X \rightarrow X / / G$ induces $T_{[x]}\left(V / / \operatorname{Stab}_{x}\right) \cong T_{[x]}(X / / G)$.

(iii) $\phi(x)=0$ and $d \phi_{x}=\mathrm{Id}$.

(iv) $T_{x} X=T_{x}\left(\mathrm{Orb}_{x}\right) \oplus T_{x} V$ with respect to the $\mathrm{Stab}_{x}$-action.

(v) $\phi$ induces $T_{[x]}\left(V / / \operatorname{Stab}_{x}\right) \cong T_{0}\left(T_{x} V / / \operatorname{Stab}_{x}\right)$.

Remark 3.15. The reader familiar with Luna's slice theorem may be wondering how Theorem 3.14 is implied. Firstly, note that $\psi$ is an étale mapping if and only if the completion of the local rings satisfy $\widehat{O}_{x} \cong \widehat{O}_{\psi(x)}$ which implies the subset of derivations are isomorphic, the latter being isomorphic to the Zariski tangent spaces. The usual Luna slice theorem implies $\phi: V / / \operatorname{Stab}_{x} \rightarrow \phi(V) / / \mathrm{Stab}_{x}$ is étale, $(G \times V) / / \operatorname{Stab}_{x} \cong U \subset X$ is saturated and open, and $V / / \operatorname{Stab}_{x} \rightarrow U / / G$ is étale. We thus respectively conclude lines (v), (iv), and (ii) in the above theorem.

Lemma 3.16. Let $G$ be a complex algebraic reductive Lie group. For any $[\rho] \in$ $\mathfrak{X}_{r}(G)$,

$$
T_{[\rho]} \mathfrak{X}_{r}(G) \cong T_{0}\left(H^{1}\left(\mathrm{~F}_{r} ; \mathfrak{g}_{\mathrm{Ad}_{\rho} \mathrm{ss}}\right) / / \operatorname{Stab}_{\rho^{\mathrm{ss}}}\right),
$$

where $\rho^{\mathrm{ss}}$ is a polystable representative from the extended orbit $[\rho]$.

Proof. Any $\rho^{\mathrm{ss}} \in[\rho]$ has a closed orbit and is a smooth point of $\mathfrak{R}_{r}(G)$, and every point $[\rho] \in \mathfrak{X}_{r}(G)$ contains such a $\rho^{\mathrm{ss}}$.

By the Luna slice theorem, there exists an algebraic set $\rho^{\mathrm{ss}} \in V_{\rho^{\mathrm{ss}}} \subset \mathfrak{R}_{r}(G)$ such that:

(i) $\operatorname{Stab}_{\rho^{\mathrm{ss}}}\left(V_{\rho^{\mathrm{ss}}}\right) \subset V_{\rho^{\mathrm{ss}}}$

(ii) With respect to the reductive action of $\operatorname{Stab}_{\rho^{s s}}$,

$$
\begin{aligned}
Z^{1}\left(\mathrm{~F}_{r} ; \mathfrak{g}_{\mathrm{Ad}_{\rho^{\mathrm{ss}}}}\right) \cong T_{\rho^{\mathrm{ss}}}\left(\mathfrak{R}_{r}(G)\right) & \cong T_{\rho^{\mathrm{ss}}}\left(\mathrm{Orb}_{\rho^{\mathrm{ss}}}\right) \oplus T_{\rho^{\mathrm{ss}}}\left(V_{\rho^{\mathrm{ss}}}\right) \\
& \cong B^{1}\left(\mathrm{~F}_{r} ; \mathfrak{g}_{\mathrm{Ad}} \rho^{\mathrm{ss}}\right) \oplus T_{\rho^{\mathrm{ss}}}\left(V_{\rho^{\mathrm{ss}}}\right),
\end{aligned}
$$

since $\rho^{\mathrm{ss}}$ is smooth.

(iii) Thus, $H^{1}\left(\mathrm{~F}_{r} ; \mathfrak{g}_{\mathrm{Ad}_{\rho} \mathrm{ss}}\right) \cong T_{\rho^{\mathrm{ss}}}\left(V_{\rho^{\mathrm{ss}}}\right)$, as $\mathrm{Stab}_{\rho^{\mathrm{ss}}-\text { spaces. }}$

(iv) $V_{\rho^{\mathrm{ss}}} \hookrightarrow \mathfrak{R}_{r}(G) \rightarrow \mathfrak{X}_{r}(G)$ induces $T_{\left[\rho^{\mathrm{ss}}\right]}\left(V_{\rho^{\mathrm{ss}}} / / \operatorname{Stab}_{\rho^{\mathrm{ss}}}\right) \cong T_{[\rho]} \mathfrak{X}_{r}(G)$.

(v) $T_{\left[\rho^{\mathrm{ss}}\right]}\left(V_{\rho^{\mathrm{ss}}} / / \mathrm{Stab}_{\rho^{\mathrm{ss}}}\right) \cong T_{0}\left(T_{\rho^{\mathrm{ss}}}\left(V_{\rho^{\mathrm{ss}}}\right) / / \mathrm{Stab}_{\rho^{\mathrm{ss}}}\right)$, since $\rho^{\mathrm{ss}}$ is smooth.

Putting these steps together we conclude

$$
T_{[\rho]} \mathfrak{X}_{r}(G) \cong T_{0}\left(T_{\rho^{\mathrm{ss}}}\left(V_{\rho^{\mathrm{ss}}}\right) / / \operatorname{Stab}_{\rho^{\mathrm{ss}}}\right) \cong T_{0}\left(H^{1}\left(\mathrm{~F}_{r} ; \mathfrak{g}_{\mathrm{Ad}_{\rho^{\mathrm{ss}}}}\right) / / \operatorname{Stab}_{\rho^{\mathrm{ss}}}\right) .
$$


Remark 3.17. Upon closer examination we find $H^{1}\left(\mathrm{~F}_{r} ; \mathfrak{g}_{\mathrm{Ad}_{\rho} \mathrm{ss}}\right) / / \mathrm{Stab}_{\rho^{\mathrm{ss}}}$ to be an étale neighborhood; that is, an algebraic set that maps, via an étale mapping, to an open set (in the ball topology) of $\mathfrak{X}_{r}(G)$ [Schwarz 2004, p. 223].

3E. The $\mathbb{C}^{*}$-action on cohomology. As we saw in Corollary 3.13 , the generic singularity will occur when $\operatorname{Stab}_{\rho}$ is the smallest possible torus group, namely $\mathbb{C}^{*}$ or $\mathbb{C}^{*} \times \mathbb{C}^{*}$, for the cases $G=\mathrm{SL}_{n}$ or $G=\mathrm{GL}_{n}$, respectively.

To study the $\mathbb{C}^{*}$-action on cohomology, the following setup will be relevant.

Fix two integers $n, k \geq 1$. Consider the vector space $\mathbb{C}^{2 n}=\mathbb{C}^{n} \times \mathbb{C}^{n}$ with variables $(\boldsymbol{z}, \boldsymbol{w})=\left(z_{1}, \ldots, z_{n}, w_{1}, \ldots, w_{n}\right)$ and the action of $\mathbb{C}^{*}$ given by

$$
\lambda \cdot(\boldsymbol{z}, \boldsymbol{w})=\left(\lambda^{k} \boldsymbol{z}, \lambda^{-k} \boldsymbol{w}\right) .
$$

Let us denote by $\mathbb{C}^{2 n} / / \mathbb{C}^{*}$ the corresponding affine GIT quotient. It is the spectrum of the ring $\mathbb{C}[\boldsymbol{z}, \boldsymbol{w}]^{\mathbb{C}^{*}}$ of polynomial invariants under this action. To describe this ring, let

$$
p(z, w)=z_{1}^{a_{1}} \cdots z_{n}^{a_{n}} w_{1}^{b_{1}} \cdots w_{n}^{b_{n}}
$$

be a monomial, with $a_{i}, b_{i} \in \mathbb{N}$, and define

$$
\partial p:=\sum_{j=1}^{n} a_{j}-b_{j} .
$$

Any polynomial invariant under the action is a sum of monomials $p$ such that $\partial p=0$. Considering the monomials with smallest degree, we are led to conclude that

$$
\mathbb{C}[\boldsymbol{z}, \boldsymbol{w}]^{\mathbb{C}^{*}}=\mathbb{C}\left[z_{1} w_{1}, \ldots, z_{1} w_{n}, \ldots, z_{n} w_{1}, \ldots, z_{n} w_{n}\right] .
$$

Note that this shows that the quotient is independent of $k$. By viewing these $n^{2}$ generators as elements of an $n \times n$ matrix $X=\left(x_{i j}\right), x_{i j}=z_{i} w_{j}$, which necessarily has rank at most one, we conclude that this is the ring of polynomial functions on the variety $V \subset M_{n \times n}(\mathbb{C})$ of matrices of rank $\leq 1$ :

$$
\mathbb{C}[z, \boldsymbol{w}]^{\mathbb{C}^{*}}=\mathbb{C}[V] .
$$

The variety $V$ is called a determinantal variety [Harris 1992] and one can show that $\mathbb{C}[V]=\mathbb{C}\left[x_{i j}\right] / I$ where $I$ is the ideal of $2 \times 2$ minors of $X$. By simple computations, $V$ has a unique singularity, the zero matrix, which corresponds to the orbit of zero in $\mathbb{C}^{2 n}$.

Now, observe that all orbits of the action (5) are closed except those contained in

$$
Z:=\{0\} \times \mathbb{C}^{n} \cup \mathbb{C}^{n} \times\{0\},
$$


and moreover there is only one closed orbit in $Z$, which is easily seen to be the only singular point of $\mathbb{C}^{2 n} / / \mathbb{C}^{*}$. Therefore, by GIT, the quotient

$$
\left(\mathbb{C}^{2 n} \backslash Z\right) / \mathbb{C}^{*}
$$

is a geometric quotient. We summarize these results as follows.

Lemma 3.18. Let $n \geq 2$.

(i) $\mathbb{C}^{2 n} / / \mathbb{C}^{*}$ is isomorphic to the determinantal variety of $n \times n$ square matrices of rank $\leq 1$. Its unique singularity is the orbit of the origin.

(ii) $\left(\mathbb{C}^{2 n} \backslash Z\right) / \mathbb{C}^{*}$ is isomorphic to $\mathbb{C}^{*} \times \mathbb{C P}^{n-1} \times \mathbb{C} \mathrm{P}^{n-1}$.

Because of the fact that the GIT quotient is obtained from $\left(\mathbb{C}^{2 n} \backslash Z\right) / \mathbb{C}^{*}$ by adding just one point, the singular point, and because of (ii) above, we will refer to $\mathbb{C}^{2 n} / / k \mathbb{C}^{*}$ as an affine cone over $\mathbb{C P} P^{n-1} \times \mathbb{C P} P^{n-1}$, and denote it by $\mathscr{C}_{\mathbb{C}}\left(\mathbb{C} P^{n-1} \times \mathbb{C P}{ }^{n-1}\right)$. It is called the affine cone over the Segre variety in [Mukai 2003].

Now consider the following antiholomorphic involution of $\mathbb{C}^{2 n}=\mathbb{C}^{n} \times \mathbb{C}^{n}$ :

$$
j:(\boldsymbol{z}, \boldsymbol{w}) \mapsto-(\overline{\boldsymbol{w}}, \bar{z}),
$$

and consider the same action as above, but restrict it to $S^{1} \subset \mathbb{C}^{*}$. This will be relevant in the study of the compact quotients. The fixed point set of the involution $j$ is the set

$$
F:=\{(z,-\bar{z}): z \in \mathbb{C}\} \subset \mathbb{C}^{n} \times \mathbb{C}^{n},
$$

which is canonically identified with the first copy of $\mathbb{C}^{n}$ (as real vector spaces).

Lemma 3.19. (i) The $S^{1}$-action on $\mathbb{C}^{2 n}$ commutes with $j$.

(ii) The quotient $F / S^{1}$ of its restriction to $F$ is homeomorphic to a real open cone over $\mathbb{C} \mathrm{P}^{n-1}$ denoted by $\mathscr{C}\left(\mathbb{C} \mathrm{P}^{n-1}\right)$.

Proof. Proving (i) is straightforward, and we leave it to the reader.

To prove (ii) first observe that on the fixed point set, the $S^{1}$-action just gives

$$
\lambda \cdot(z,-\bar{z})=(\lambda z,-\bar{\lambda} \bar{z}), \quad \lambda \in S^{1}
$$

so we can describe it as an action of $S^{1}$ on the first copy of $\mathbb{C}^{n}$. Since the action is free except for the origin, all orbits are circles and the quotient $\mathbb{C}^{n} / S^{1}$ is the union of $\mathbb{C}^{n} \backslash\{0\} / S^{1}$ with a single point. Since $\mathbb{C}^{n} \backslash\{0\} / S^{1}$ is homeomorphic to $\left(S^{2 n-1} / S^{1}\right) \times \mathbb{R}$, we obtain that $F / S^{1}$ is the real cone over $S^{2 n-1} / S^{1}$, the latter being well known to be $\mathbb{C P}^{n-1}$.

These singularity types will be encountered in $\mathrm{SL}_{n}$ and $\mathrm{SU}_{n}$ - character varieties. In fact, the same singularities will also appear in $\mathrm{GL}_{n}$ and $\mathrm{U}_{n}$-character varieties, because the actions in these cases are very similar.

Indeed one can easily show the following: 
Proposition 3.20. Let $n \geq 2$. Let $T=\mathbb{C}^{*} \times \mathbb{C}^{*}$ act on a vector space $V=\mathbb{C}^{2 n}=$ $\mathbb{C}^{n} \times \mathbb{C}^{n}$ as follows:

$$
(\lambda, \mu) \cdot(z, w)=\left(\lambda \mu^{-1} z, \mu \lambda^{-1} w\right) .
$$

Then, $\mathbb{C}^{2 n} / / T$ is isomorphic to $\mathbb{C}^{2 n} / / 2 \mathbb{C}^{*}$. In particular, as before, this quotient is the determinantal variety of $n \times n$ square matrices of rank $\leq 1$, which has dimension $2 n-1$. Its unique singularity is the orbit of the origin.

Proof. We just need to argue, as before, that the invariant polynomials are generated by the same monomials, those of the form $z_{j} w_{k}$, for any indices $j, k \in\{1, \ldots, n\}$, so they form an $n \times n$ matrix with rank one.

Finally, note that for $n=1$ we get a smooth variety: $\mathbb{C}^{2} / / 2 \mathbb{C}^{*} \cong \mathbb{C}$.

\section{F. Proof of Theorem 1.1, Case 1: $\mathrm{GL}_{n}$ or $\mathrm{SL}_{n}$.}

Theorem 3.21. Let $r, n \geq 2$ and $G=\mathrm{GL}_{n}$ or $\mathrm{SL}_{n}$. Then $\mathfrak{X}_{r}(G)^{\text {sing }}=\mathfrak{X}_{r}(G)^{\text {red }}$ if and only if $(r, n) \neq(2,2)$.

Remark 3.22. If $n=1$ the statement is vacuously true since in these cases there are no reducibles, nor are there singularities. We have already seen that there are smooth reducibles in the cases $r=1, n \geq 2$, and $(r, n)=(2,2)$ since there always exist reducibles in these cases and the entire moduli spaces are smooth.

Proof. Let $G=\mathrm{GL}_{n}$. By Lemma 3.7 it is enough to show $\mathfrak{X}_{r}(G)^{\text {red }} \subset \mathfrak{X}_{r}(G)^{\text {sing }}$.

Let $\rho \in U_{r, n} \subset \mathfrak{R}_{r}(G)^{\text {red }}$ be of reduced type $\left[n_{1}, n_{2}\right]$ with $n_{1}, n_{2}>0$ and $n=$ $n_{1}+n_{2}$ (see Definition 3.10) and write it in the form

$$
\rho=\rho_{1} \oplus \rho_{2}=\left(\begin{array}{cc}
\vec{X} & \overrightarrow{0}_{n_{1} \times n_{2}} \\
\overrightarrow{0}_{n_{2} \times n_{1}} & \vec{Y}
\end{array}\right),
$$

where $\vec{X}=\left(X_{1}, \ldots, X_{r}\right) \in M_{n_{1} \times n_{1}}^{r}$ and $\vec{Y}=\left(Y_{1}, \ldots, Y_{r}\right) \in M_{n_{2} \times n_{2}}^{r}$ and $\overrightarrow{0}_{k \times l}=$ $\left(0_{k \times l}, \ldots, 0_{k \times l}\right)$ where $0_{k \times l}$ is the $k$ by $l$ matrix of zeros and the vector has $r$ entries. Recall that these representations form a dense set in $\mathfrak{X}_{r}(G)^{\text {red }}$, by Lemma 3.12.

Let $\operatorname{diag}\left(a_{1}, \ldots, a_{n}\right)$ be an $n \times n$ matrix whose $(i, j)$-entry is 0 if $i \neq j$ and is equal to $a_{i}$ otherwise. Then $\operatorname{Stab}_{\rho}=\mathbb{C}^{*} \times \mathbb{C}^{*}$ is given by

$$
\operatorname{diag}(\underbrace{\lambda, \ldots, \lambda}_{n_{1}}, \overbrace{\mu, \ldots, \mu}^{n_{2}}) .
$$

We note that the action of the center is trivial so we often consider the stabilizer with respect to the action of $G$ modulo its center. 
Then the cocycles satisfy

$$
\begin{aligned}
Z^{1}\left(\mathrm{~F}_{r} ; \mathrm{Ad}_{\rho}\right) & \cong \mathfrak{g}^{r} \\
& =\left\{\left(\begin{array}{cc}
\vec{A} & \vec{B} \\
\vec{C} & \vec{D}
\end{array}\right) \mid \vec{A} \in M_{n_{1} \times n_{1}}^{r}, \vec{B} \in M_{n_{1} \times n_{2}}^{r}, \vec{C} \in M_{n_{2} \times n_{1}}^{r}, \vec{D} \in M_{n_{2} \times n_{2}}^{r}\right\},
\end{aligned}
$$

which has dimension $n^{2} r$ since it is the tangent space to a representation and the representation variety is smooth. The coboundaries are given by

$$
\begin{aligned}
B^{1}\left(\mathrm{~F}_{r} ; \operatorname{Ad}_{\rho}\right) & \cong\left\{\left(\begin{array}{cc}
A & B \\
C & D
\end{array}\right)-\left(\begin{array}{cc}
\vec{X} & \overrightarrow{0}_{n_{1} \times n_{2}} \\
\overrightarrow{0}_{n_{2} \times n_{1}} & \vec{Y}
\end{array}\right)\left(\begin{array}{cc}
A & B \\
C & D
\end{array}\right)\left(\begin{array}{cc}
\vec{X}^{-1} & \overrightarrow{0}_{n_{1} \times n_{2}} \\
\overrightarrow{0}_{n_{2} \times n_{1}} & \vec{Y}^{-1}
\end{array}\right)\right\} \\
& \cong\left\{\left(\begin{array}{cc}
A & B \\
C & D
\end{array}\right)-\left(\begin{array}{cc}
\vec{X} A \vec{X}^{-1} & \vec{X} B \vec{Y}^{-1} \\
\vec{Y} C \vec{X}^{-1} & \vec{Y} D \vec{Y}^{-1}
\end{array}\right)\right\},
\end{aligned}
$$

for a fixed element $\left(\begin{array}{ll}A & B \\ C & D\end{array}\right) \in \mathfrak{g}$. This has dimension $n^{2}-2$ since it is the tangent space to the $G$-orbit of $\rho$ which has dimension equal to that of the group minus its stabilizer.

Thus with respect to the torus action,

$$
H^{1}\left(\mathrm{~F}_{r} ; \operatorname{Ad}_{\rho}\right) \cong H^{1}\left(\mathrm{~F}_{r} ; \operatorname{Ad}_{\rho_{1}}\right) \oplus H^{1}\left(\mathrm{~F}_{r} ; \operatorname{Ad}_{\rho_{2}}\right) \oplus W,
$$

where $W$ exists since the torus action is reductive. By considering the Euler characteristic, one has that

$$
\operatorname{dim}_{\mathbb{C}} H^{0}\left(\mathrm{~F}_{r} ; \operatorname{Ad}_{\rho}\right)-\operatorname{dim}_{\mathbb{C}} H^{1}\left(\mathrm{~F}_{r} ; \operatorname{Ad}_{\rho}\right)=(1-r) \operatorname{dim}_{\mathbb{C}} \mathfrak{g l}(n, \mathbb{C}) .
$$

Then since $H^{0}\left(\mathrm{~F}_{r} ; \operatorname{Ad}_{\rho}\right)=Z^{0}\left(\mathrm{~F}_{r} ; \operatorname{Ad}_{\rho}\right)$ is the centralizer in $\mathfrak{g}$ of the image of $\rho$, we calculate

$$
\begin{aligned}
\operatorname{dim}_{\mathbb{C}} H^{1}\left(\mathrm{~F}_{r} ; \operatorname{Ad}_{\rho}\right) & =n^{2}(r-1)+2, \\
\operatorname{dim}_{\mathbb{C}} H^{1}\left(\mathrm{~F}_{r} ; \operatorname{Ad}_{\rho_{i}}\right) & =n_{i}^{2}(r-1)+1, \quad i=1,2 .
\end{aligned}
$$

This then implies $\operatorname{dim}_{\mathbb{C}} H^{1}\left(\mathrm{~F}_{r} ; \operatorname{Ad}_{\rho}\right) / /\left(\mathbb{C}^{*} \times \mathbb{C}^{*}\right)=n^{2}(r-1)+1=\operatorname{dim}_{\mathbb{C}} \mathfrak{X}_{r}(G)$, since the diagonal of the $\mathbb{C}^{*} \times \mathbb{C}^{*}$-action is the center which acts trivially. We conclude that

$$
\operatorname{dim}_{\mathbb{C}} W=\left(n^{2}-n_{1}^{2}-n_{2}^{2}\right)(r-1)=2 n_{1} n_{2}(r-1) .
$$

Explicitly, the $\operatorname{Stab}_{\rho}$ action on $H^{1}\left(\mathrm{~F}_{r} ; \mathrm{Ad}_{\rho}\right)$ is given by

$$
\operatorname{diag}(\underbrace{\lambda, \ldots, \lambda,}_{n_{1}} \overbrace{\mu, \ldots, \mu}^{n_{2}}) \cdot\left[\left(\begin{array}{cc}
\vec{A} & \vec{B} \\
\vec{C} & \vec{D}
\end{array}\right)\right] \mapsto\left[\left(\begin{array}{cc}
\vec{A} & \lambda \vec{B} \mu^{-1} \\
\mu \vec{C} \lambda^{-1} & \vec{D}
\end{array}\right)\right]
$$

which respects representatives up to coboundary. 
So, the action on $H^{1}\left(\mathrm{~F}_{r} ; \operatorname{Ad}_{\rho_{1}}\right) \oplus H^{1}\left(\mathrm{~F}_{r} ; \operatorname{Ad}_{\rho_{2}}\right)$ is trivial (but not so on $W$ ) and we conclude

$$
H^{1}\left(\mathrm{~F}_{r} ; \operatorname{Ad}_{\rho}\right) /\left(\mathbb{C}^{*} \times \mathbb{C}^{*}\right) \cong H^{1}\left(\mathrm{~F}_{r} ; \operatorname{Ad}_{\rho_{1}}\right) \oplus H^{1}\left(\mathrm{~F}_{r} ; \operatorname{Ad}_{\rho_{2}}\right) \oplus\left(W / /\left(\mathbb{C}^{*} \times \mathbb{C}^{*}\right)\right) .
$$

Therefore, by Proposition 3.20, we have established that 0 is a singularity (solution to the generators of the singular locus) of $W /\left(\mathbb{C}^{*} \times \mathbb{C}^{*}\right)$ which then implies it is a singularity to $H^{1}\left(\mathrm{~F}_{r} ; \operatorname{Ad}_{\rho}\right) / /\left(\mathbb{C}^{*} \times \mathbb{C}^{*}\right)$ (whenever $\operatorname{dim}_{\mathbb{C}} W>2$ ) which then in turn implies any $\rho \in U_{r, n}$ is a singularity in $\mathfrak{X}_{r}(G)$ by Lemma 3.16 (note $\rho=\rho^{\text {ss }}$ here). $U_{r, n}$ is dense in $\mathfrak{X}_{r}\left(\mathrm{GL}_{n}\right)^{\text {red }}$ by Lemma 3.12. Then Lemma 3.9 applies to show that $\mathfrak{X}_{r}\left(\mathrm{GL}_{n}\right)^{\text {sing }}=\mathfrak{X}_{r}\left(\mathrm{GL}_{n}\right)^{\text {red }}$ whenever $\operatorname{dim}_{\mathbb{C}} W=2 n_{1} n_{2}(r-1)>2$; that is, whenever $(r, n) \neq(2,2)$.

Now let $[\rho] \in \mathfrak{X}_{r}\left(\mathrm{SL}_{n}\right)$. Then it is easy to see that $[\rho] \in \mathfrak{X}_{r}\left(\mathrm{SL}_{n}\right)^{\text {red }}$ if and only if $[\rho] \in \mathfrak{X}_{r}\left(\mathrm{GL}_{n}\right)^{\text {red }}$. Then Corollary 2.7 and the previously established case together imply $\mathfrak{X}_{r}\left(\mathrm{SL}_{n}\right)^{\text {red }}=\mathfrak{X}_{r}\left(\mathrm{SL}_{n}\right)^{\text {sing }}$.

This finishes the proof of Theorem 1.1 for the groups $\mathrm{SL}_{n}$ and $\mathrm{GL}_{n}$.

Remark 3.23. We note that the cohomology decomposition used in the proof depends on the decomposition of $\rho$. For instance, in the $2 \times 2$ determinant 1 case, the reducible representation takes values in $\mathrm{SL}_{1} \times \mathrm{GL}_{1}=\mathbb{C}^{0} \times \mathbb{C}^{*}$, where $\mathbb{C}^{0}$ is a point. Then by Lemma 3.18:

$$
\begin{aligned}
H^{1}\left(\mathrm{~F}_{r}, \operatorname{Ad}_{\rho}\right) / / \mathbb{C}^{*} & \cong H^{1}\left(\mathrm{~F}_{r}, \operatorname{Ad}_{\rho_{1}}\right) \oplus H^{1}\left(\mathrm{~F}_{r}, \operatorname{Ad}_{\rho_{2}}\right) \oplus\left(W / / \mathbb{C}^{*}\right) \\
& \cong \mathbb{C}^{0} \times \mathbb{C}^{r} \times\left(\left(\mathbb{C}^{2 r} / \mathbb{C}^{2}\right) / / \mathbb{C}^{*}\right) \\
& \cong \mathbb{C}^{r} \times \mathbb{C}^{2 r-2} / / 2 \mathbb{C}^{*} \\
& \cong \mathbb{C}^{r} \times \mathscr{C}_{\mathbb{C}}\left(\mathbb{C} P^{r-2} \times \mathbb{C} P^{r-2}\right) .
\end{aligned}
$$

Remark 3.24. The proof above works directly, with suitable modifications for the case $G=\mathrm{SL}_{n}$. For instance the action of the stabilizer in this case is $\operatorname{Stab}_{\rho}=\mathbb{C}^{*}$ given by

$$
\operatorname{diag}(\underbrace{\lambda, \ldots, \lambda,}_{n_{1}} \overbrace{\mu, \ldots, \mu}^{n_{2}}),
$$

where $\lambda^{n_{1}} \mu^{n_{2}}=1$ which is equivalent to $\mu=\lambda^{\frac{-n_{1}}{n_{2}}}$. The cocycles satisfy

$$
\begin{aligned}
& Z^{1}\left(\mathrm{~F}_{r} ; \operatorname{Ad}_{\rho}\right) \\
& \cong \mathfrak{g}^{r}=\left\{\left(\begin{array}{cc}
\vec{A} & \vec{B} \\
\vec{C} & \vec{D}
\end{array}\right) \mid \vec{A} \in M_{n_{1} \times n_{1}}^{r}, \vec{B} \in M_{n_{1} \times n_{2}}^{r}, \vec{C} \in M_{n_{2} \times n_{1}}^{r}, \vec{D} \in M_{n_{2} \times n_{2}}^{r},\right. \\
& \left.\operatorname{tr}\left(A_{i}\right)=-\operatorname{tr}\left(D_{i}\right), 1 \leq i \leq r\right\},
\end{aligned}
$$

which has dimension $\left(n^{2}-1\right) r$. The rest carries over without significant change. 
Remark 3.25. Similar results for the moduli of tuples of generic matrices have been obtained in [Le Bruyn and Procesi 1987], and with respect to the moduli of vector bundles similar results have been obtained in [Laszlo 1996].

3G. Proof of Theorem 1.1, Case 2: $\mathrm{SU}_{n}$ or $\mathrm{U}_{n}$. Let $K=\mathrm{SU}_{n}$ or $\mathrm{U}_{n}$ and let $\mathfrak{k}$ be its Lie algebra in either case.

The tangent space at a point $[\rho] \in \mathfrak{X}_{r}(K)$ is defined from the semialgebraic structure; that is, any real semialgebraic set has a well-defined coordinate ring which allows one to define the Zariski tangent space as we did at the start of this section [Bochnak et al. 1998]. At smooth points this corresponds to the usual tangent space defined by differentials. It is not hard to see that the semialgebraic set $\mathfrak{X}_{r}(K)$ is a subset of the real points of $\mathfrak{X}_{r}\left(K_{\mathbb{C}}\right)$. Then, the Zariski tangent space of $\mathfrak{X}_{r}(K)$ at $[\rho], T_{[\rho]}\left(\mathfrak{X}_{r}(K)\right)$, consists of the real points of the complex Zariski tangent space $T_{[\rho]}\left(\mathfrak{X}_{r}\left(K_{\mathbb{C}}\right)\right)$.

As is true for $K_{\mathbb{C}}$-representations, we define a $K$-representation to be irreducible if it does not admit any proper (nontrivial) invariant subspaces with respect to the standard action on $\mathbb{C}^{n}$. As with $K_{\mathbb{C}}$-valued representations, we call a $K$-valued representation reducible if it is not irreducible.

Lemma 3.26. $\mathfrak{X}_{r}\left(K_{\mathbb{C}}\right)^{\text {red }} \cap \mathfrak{X}_{r}(K)=\mathfrak{X}_{r}(K)^{\text {red }}$.

Proof. First note that $\mathfrak{X}_{r}(K) \subset \mathfrak{X}_{r}\left(K_{\mathbb{C}}\right)$ (see [Florentino and Lawton 2009]). So it suffices to prove that every $K$-valued representation is $K$-conjugate to a reducible representation if and only if it is $K_{\mathbb{C}}$-conjugate to a reducible representation.

Let $\rho$ be a $K$-representation and suppose that it is $K$-conjugate to a representation that admits a nontrivial proper invariant subspace of $\mathbb{C}^{n}$, then since $K \subset K_{\mathbb{C}}$ it

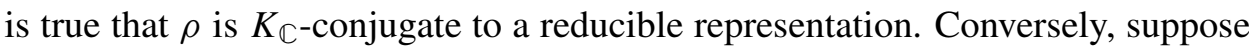
that a $K$-representation $\rho$ is $K_{\mathbb{C}}$-conjugate to a reducible representation. However, conjugating by $K_{\mathbb{C}}$ is simply a change-of-basis, and such a change-of-basis is always possible by conjugating by $K$ by using the Gram-Schmidt algorithm.

Lemma 3.27. $\mathfrak{X}_{r}\left(K_{\mathbb{C}}\right)^{\text {sing }} \cap \mathfrak{X}_{r}(K)=\mathfrak{X}_{r}(K)^{\text {sing }}$

Proof. Let

$$
[\rho] \in \mathfrak{X}_{r}(K) \subset \mathfrak{X}_{r}\left(K_{\mathbb{C}}\right) .
$$

Then $[\rho] \in \mathfrak{X}_{r}(K)^{\text {sing }}$ if and only if $\operatorname{dim}_{\mathbb{R}} T_{[\rho]} \mathfrak{X}_{r}(K)=\operatorname{dim}_{\mathbb{C}} T_{[\rho]} \mathfrak{X}_{r}\left(K_{\mathbb{C}}\right)$ exceeds $\operatorname{dim}_{\mathbb{R}} \mathfrak{X}_{r}(K)=\operatorname{dim}_{\mathbb{C}} \mathfrak{X}_{r}\left(K_{\mathbb{C}}\right)$, the latter occurring if and only if $[\rho] \in \mathfrak{X}_{r}\left(K_{\mathbb{C}}\right)^{\text {sing }}$.

The last case to consider to finish the proof of Theorem 1.1 is $\mathfrak{X}_{r}(K)$ in terms of $\mathrm{SU}_{n}$ and $\mathrm{U}_{n}$.

Theorem 3.28. Let $K$ be either $\mathrm{U}_{n}$ or $\mathrm{SU}_{n}$. Then $\mathfrak{X}_{r}(K)^{\mathrm{red}}=\mathfrak{X}_{r}(K)^{\text {sing }}$ if

$$
\mathfrak{X}_{r}\left(K_{\mathbb{C}}\right)^{\text {red }}=\mathfrak{X}_{r}\left(K_{\mathbb{C}}\right)^{\text {sing }} .
$$


Proof. This follows directly by Lemmas 3.26 and 3.27.

Since we have already established in Theorem 3.21 that, for $r, n \geq 2$ and $K \in\left\{\mathrm{U}_{n}, \mathrm{SU}_{n}\right\}$, we have $\mathfrak{X}_{r}\left(K_{\mathbb{C}}\right)^{\text {red }}=\mathfrak{X}_{r}\left(K_{\mathbb{C}}\right)^{\text {sing }}$ if and only if $(r, n) \neq(2,2)$, Theorem 3.28 is enough to finish the proof of Theorem 1.1.

3H. Iterative reducibles and the singular stratification. As above let $K$ be either $\mathrm{U}_{n}$ or $\mathrm{SU}_{n}$ and $G=K_{\mathbb{C}}$, and let the $N$-th singular stratum be defined by

$$
\operatorname{Sing}_{N}\left(\mathfrak{X}_{r}(G)\right)=\left(\cdots\left(\left(\mathfrak{X}_{r}(G)\right)^{\text {sing }}\right)^{\text {sing } \cdots}\right)^{\text {sing }},
$$

which is well-defined since each singular locus is a variety and as such has a singular locus itself.

The $N$-th level reducibles

$$
\operatorname{Red}_{N}\left(\mathfrak{X}_{r}(G)\right)=\left(\cdots\left(\left(\mathfrak{X}_{r}(G)\right)^{\text {red }}\right)^{\text {red } \cdots}\right)^{\text {red }}
$$

is defined inductively in the following way.

Let $\operatorname{Red}_{1}\left(\mathfrak{X}_{r}(G)\right)=\mathfrak{X}_{r}(G)^{\text {red }}$. For $k \geq 1$ define $\operatorname{Red}_{k}\left(\mathfrak{X}_{r}(G)\right)^{(k+1)}$ to be the set of $\rho \in \operatorname{Red}_{k}\left(\mathfrak{X}_{r}(G)\right)$ which is minimally reducible, that is has a decomposition into irreducible subrepresentations that has minimal summands. We define $\operatorname{Red}_{k+1}\left(\mathfrak{X}_{r}(G)\right)=\operatorname{Red}_{k}\left(\mathfrak{X}_{r}(G)\right)-\operatorname{Red}_{k}\left(\mathfrak{X}_{r}(G)\right)^{(k+1)}$ to be the complement of $\operatorname{Red}_{k}\left(\mathfrak{X}_{r}(G)\right)^{(k+1)}$ in $\operatorname{Red}_{k}\left(\mathfrak{X}_{r}(G)\right)$. Thus, $\operatorname{Red}_{1}\left(\mathfrak{X}_{r}(G)\right)^{(2)}$ is always the reducibles that have exactly 2 irreducible subrepresentations - these are exactly the ones we considered in the proof of Theorem 3.21. More generally, $\operatorname{Red}_{k}\left(\mathfrak{X}_{r}(G)\right)^{(k+1)}$ are the representations which decompose into exactly $k+1$ irreducible subrepresentations. For example, $\operatorname{Red}_{2}\left(\mathfrak{X}_{r}\left(\mathrm{SL}_{3}\right)\right)$ are the representations conjugate to a representation that has its semisimplification diagonal, and $\operatorname{Red}_{3}\left(\mathfrak{X}_{r}\left(\mathrm{SL}_{3}\right)\right)=\varnothing$.

Likewise we have $\operatorname{Red}_{N}\left(\mathfrak{X}_{r}(K)\right)$ and $\operatorname{Sing}_{N}\left(\mathfrak{X}_{r}(K)\right)$.

Theorem 3.29. Let $r, n \geq 2$ and $(r, n) \neq(2,2)$. If $N \geq 1$, then

$$
\operatorname{Sing}_{N}\left(\mathfrak{X}_{r}(G)\right) \cong \operatorname{Red}_{N}\left(\mathfrak{X}_{r}(G)\right) \text { and } \operatorname{Sing}_{N}\left(\mathfrak{X}_{r}(K)\right) \cong \operatorname{Red}_{N}\left(\mathfrak{X}_{r}(K)\right) \text {. }
$$

The result follows by induction on the irreducible block forms and observing that each block form now corresponds to $\mathrm{GL}_{k}$, or $\mathrm{U}_{k}$ in the compact cases.

\section{I. Remarks about other groups.}

3I.1. General reductive groups. Let $G$ be a reductive complex algebraic group. It can be shown [Sikora 2012] that the definition given before of an irreducible representation $\rho: \Gamma \rightarrow G$ corresponds exactly to the quotient group $\operatorname{Stab}_{\rho} / Z(G)$ being finite.

Proposition 3.30. If the adjoint action of $\rho$ is irreducible on $\mathfrak{g}$, then $[\rho]$ is smooth in $\mathfrak{X}_{r}(G)$. 
Proof. If $\mathrm{Ad}_{\rho}$ is irreducible, then Stab $\rho_{\rho^{\text {ss }}}$ is central and so Stab $\rho_{\rho^{\text {ss }}}$ acts trivially on $H^{1}\left(\mathrm{~F}_{r} ; \mathfrak{g}_{\mathrm{Ad}_{\rho} \mathrm{ss}}\right)$. Hence 0 is not in the Jacobian ideal of $H^{1}\left(\mathrm{~F}_{r} ; \mathfrak{g}_{\mathrm{Ad}} \mathrm{ss}^{\mathrm{ss}}\right) / / \mathrm{Stab}_{\rho^{\mathrm{ss}}}$. So, by Lemma 3.16, $[\rho]$ is smooth in $\mathfrak{X}_{r}(G)$.

From the proof of Proposition 3.30, we obtain:

Corollary 3.31. Let $G$ be a complex reductive algebraic group and $\rho \in \mathfrak{R}_{r}(G)$ is irreducible with central stabilizer. Then $[\rho]$ is smooth in $\mathfrak{X}_{r}(G)$.

A representation satisfying the conditions of this corollary is called good. In other words, $\rho \in \mathfrak{R}_{r}(G)^{s}$ is good if and only if $\operatorname{Stab}_{\rho} / Z(G)$ is trivial. Letting $\mathfrak{R}_{r}(G)^{\text {good }}$ be the open subset of good representations, it easily follows that

$$
\mathfrak{X}_{r}(G)^{\text {good }}:=\mathfrak{R}_{r}(G)^{\text {good }} / G \subset \mathfrak{X}_{r}(G)^{s} \subset \mathfrak{X}_{r}(G)
$$

is always a smooth manifold.

[Heusener and Porti 2004] shows that our main theorem, i.e.,

$$
\mathfrak{X}_{r}(G)^{\text {red }}=\mathfrak{X}_{r}(G)^{\text {sing }},
$$

is not true for all reductive Lie groups $G$ and free groups $\mathrm{F}_{r}$ since for $\mathrm{PSL}_{2}$ there are irreducible representations which are singular. The issue is that the stabilizer of an irreducible representation, modulo the center of $G$, may not be trivial in general. This is not an issue for $\mathrm{GL}_{n}$ or $\mathrm{SL}_{n}$ since Lemma 3.5 shows the action is free on the set of irreducibles; that is, in these cases a representation is good if and only if it is irreducible.

Let $\mathrm{O}_{n}$ be the group of $n \times n$ complex orthogonal matrices, and let $\mathrm{Sp}_{2 n}$ be the group of $2 n \times 2 n$ complex symplectic matrices.

Proposition 3.32. There exists irreducible representations $\rho: \mathrm{F}_{r} \rightarrow G$ for $G$ any of $\mathrm{O}_{n}, \mathrm{PSL}_{n}$, and $\mathrm{Sp}_{2 n}$ such that $\rho$ is not good.

Proof. It is sufficient in each case to find, for some $n$, a nonparabolic subgroup of $G$ whose centralizer contains a noncentral element.

First consider a $\mathrm{SL}_{2}$-representation $\rho$ contained in the subgroup of diagonal and antidiagonal matrices (containing at least one nondiagonal element and one noncentral element). Then $\mathrm{Stab}_{\rho} / Z\left(\mathrm{SL}_{2}\right)$ is trivial, and so such a representation is irreducible. However $\rho$ also determines an irreducible $\mathrm{PSL}_{2}$-valued representation consisting of diagonal and antidiagonal matrices. However, its stabilizer now contains $\left(\begin{array}{cc}i & 0 \\ 0 & -i\end{array}\right)$, since this element acts as scalar multiplication by -1 on the antidiagonal components and trivially on the diagonal components, so the action is trivial for $\mathrm{PSL}_{2}$-representations but nontrivial for $\mathrm{SL}_{2}$-representations. This element is not central in $\mathrm{SL}_{2}$. Thus $\rho$ defines an irreducible representation into $\mathrm{PSL}_{2}$ that has finite noncentral stabilizer, and thus is not good.

For $\mathrm{O}_{n}$ representations consider any representation whose image consists of all 
matrices of the form

$$
\left\{\left(\begin{array}{cccc} 
\pm 1 & 0 & 0 & 0 \\
0 & \pm 1 & 0 & 0 \\
0 & 0 & \ddots & 0 \\
0 & 0 & 0 & \pm 1
\end{array}\right)\right\}
$$

One easily computes that the stabilizer is finite and not trivial and thus they are irreducible with $\mathrm{Stab}_{\rho} / Z\left(\mathrm{O}_{n}\right)$ not trivial and thus are not good.

For $\mathrm{Sp}_{2 n}$ representations we can likewise find examples like the following for $n=2$. Let the representation have its image generated by

$$
\left\{ \pm\left(\begin{array}{rrrr}
0 & 0 & 0 & 1 \\
0 & 0 & 1 & 0 \\
0 & -1 & 0 & 0 \\
-1 & 0 & 0 & 0
\end{array}\right), \pm\left(\begin{array}{rrrr}
i & 0 & 0 & 0 \\
0 & -i & 0 & 0 \\
0 & 0 & i & 0 \\
0 & 0 & 0 & -i
\end{array}\right), \pm\left(\begin{array}{rrrr}
0 & 0 & 0 & -1 \\
0 & 0 & 1 & 0 \\
0 & -1 & 0 & 0 \\
1 & 0 & 0 & 0
\end{array}\right)\right\}
$$

We get a subgroup of order 16 with finite stabilizer; as such, this group is an irreducible with finite noncentral stabilizer. Again we see that $\operatorname{Stab}_{\rho} / Z\left(\mathrm{Sp}_{2 n}\right)$ is not trivial and thus this representation is not good.

Remark 3.33. In the case of $\mathrm{PSL}_{2}$ representations (and consequently for $\mathrm{SL}_{2}$ valued representations) there are irreducible representations that act reducibly on $\mathfrak{g}$. However, for $\mathrm{PSL}_{2}$ these are singular points, but for $\mathrm{SL}_{2}$ they are smooth. This shows that Ad-reducibility does not imply nonsmoothness in general. In fact, in $\mathfrak{X}_{2}\left(\mathrm{PSL}_{2}\right)$ there are simultaneously reducibles that are smooth points and irreducibles that are singular. See [Heusener and Porti 2004].

Conjecture 3.34. Let $G$ be a complex reductive algebraic group, and suppose $r \geq 3$. Then $\mathfrak{X}_{r}(G)^{\text {red }} \subset \mathfrak{X}_{r}(G)^{\text {sing }}$, and if $G$ is semisimple equality holds if and only if $G$ is a Cartesian product of $\mathrm{SL}_{n}$ 's.

We leave the exploration of this interesting conjecture and the description of singular irreducibles to future work.

3I.2. What if $\Gamma$ is not free? One may wonder what the relationships exist, if any, between reducible representations and singular points in $\mathfrak{X}_{\Gamma}(G)$ for a general finitely generated group $\Gamma$.

With a given presentation of $\Gamma$ as $\Gamma=\left\langle x_{1}, \ldots, x_{r} \mid r_{1}, \ldots, r_{k}\right\rangle$ we can naturally associate the canonical epimorphism $\mathrm{F}_{r} \rightarrow \Gamma=F_{r} /\left\langle r_{1}, \ldots, r_{k}\right\rangle$ which induces the inclusion $\mathfrak{X}_{\Gamma}(G) \subset \mathfrak{X}_{\mathrm{F}_{r}}(G)$ providing $\mathfrak{X}_{\Gamma}(G)$ with the structure of an affine subvariety. As such, $\rho$ is irreducible (resp. completely reducible) in $\mathfrak{X}_{\Gamma}(G)$ if and only if $\rho$ is irreducible (resp. completely reducible) in $\mathfrak{X}_{\mathrm{F}_{r}}(G)$.

However, the notion of singularity is very far from being well behaved: 
(i) If $\Gamma$ is free abelian then all representations are reducible and thus the singularities cannot equal the reducibles since the singularities are a proper subset. So reducibles can be smooth; in fact this example shows all smooth points can be reducible.

(ii) The irreducibles are not generally all smooth in the representation variety let alone in the quotient variety; see [Sikora 2012, Example 38]. Such representations can project to singular points in the quotient (as one might hope is the general situation). Therefore, there can be representations in $\mathfrak{X}_{\Gamma}(G)^{\text {sing }} \subset \mathfrak{X}_{\Gamma}(G) \subset \mathfrak{X}_{\mathrm{F}_{r}}(G)$ which are smooth in $\mathfrak{X}_{\mathrm{F}_{r}}(G)$.

(iii) Singularities in the quotient do not necessarily arise from singularities in the representation space. For example, if $\Gamma$ is the fundamental group of a genus 2 surface there exist representations in $\mathfrak{R}_{\Gamma}\left(\mathrm{SU}_{2}\right)$ that are singular but the quotient $\mathfrak{X}_{\Gamma}\left(\mathrm{SU}_{2}\right) \approx \mathbb{C} \mathrm{P}^{3}$ is smooth. See [Narasimhan and Seshadri 1965; Narasimhan and Ramanan 1969].

(iv) Lemma 3.16 and its generalizations [Sikora 2012] do not necessarily apply in general.

Therefore, when $\Gamma$ is not free there is little one can say in general.

\section{Local structure and classification of manifold cases}

Having completed the proof of Theorem 1.1, we now move on to prove Theorem 1.2. As stated earlier, in [Bratholdt and Cooper 2001] it is established that $\mathfrak{X}_{r}\left(\mathrm{SU}_{2}\right)$ are not topological manifolds when $r \geq 4$. They compute explicit examples where the representations (abelian, nontrivial) are contained in a neighborhood homeomorphic to $\mathscr{C}\left(\mathbb{C P}^{r-2}\right) \times \mathbb{R}^{r}$, where $\mathscr{C}(X)=(X \times[0,1)) /(X \times\{0\})$ is the real open cone over a topological space $X$. From this characterization, simple arguments imply that $\mathfrak{X}_{r}\left(\mathrm{SU}_{2}\right)$ is not a manifold for $r \geq 4$. It is also a consequence of the following criterion, which will be useful later.

Lemma 4.1. Let $X$ be a manifold of dimension $n$ and let $d \geq 0$. If $\mathscr{b}(X) \times \mathbb{R}^{d}$ is Euclidean (i.e, homeomorphic to $\mathbb{R}^{d+n+1}$ ) then $X$ is homotopically equivalent to $S^{n}$ (a sphere of dimension $n$ ). Also, if $\mathscr{C}(X) \times \mathbb{R}^{d}$ is half-Euclidean (i.e, homeomorphic to a closed half-space in $\mathbb{R}^{d+n+1}$ ) then $X$ is homotopically equivalent to either a point or $S^{n}$.

Proof. Let $p$ be the cone point of $\mathscr{C}(X)$. Using the natural deformation retraction from $\mathscr{b}(X)-\{p\}$ to $X$, we see that

$$
\mathscr{C}(X) \times \mathbb{R}^{d}-\left(\{p\} \times \mathbb{R}^{d}\right)=X \times(0,1) \times \mathbb{R}^{d} \simeq X,
$$

where $Y \simeq X$ symbolizes $Y$ being homotopic to $X$. On the other hand, if $\mathscr{C}(X) \times \mathbb{R}^{d}=$ $\mathbb{R}^{n+d+1}$ then $\mathscr{C}(X) \times \mathbb{R}^{d}-\left(\{p\} \times \mathbb{R}^{d}\right)=\mathbb{R}^{n+d+1}-\mathbb{R}^{d} \simeq S^{n}$. 
The other statement follows in a similar fashion if the cone point is not on the boundary of the half-space. Otherwise, $\{p\} \times \mathbb{R}^{d}$ is contained in the boundary so extracting it results in a contractible space.

4A. $\mathfrak{X}_{r}\left(\mathrm{SU}_{n}\right)$ and $\mathfrak{X}_{r}\left(\mathrm{U}_{n}\right)$. In this subsection we establish the compact cases of Theorem 1.2.

Let $K=\mathrm{SU}_{n}$ and let $\mathfrak{k}$ be its Lie algebra. Let $d_{r, n}=\left(n^{2}-1\right)(r-1)=$ $\operatorname{dim}_{\mathbb{C}} \mathfrak{X}_{r}(G)=\operatorname{dim}_{\mathbb{R}} \mathfrak{X}_{r}(K)$. Whenever $\mathfrak{X}_{r}(K)$ is not a topological manifold, there exists a point $[\rho] \in \mathfrak{X}_{r}(K)$ and a neighborhood $\mathcal{N}$ containing $[\rho]$ that is not locally homeomorphic to $\mathbb{R}^{d_{r, n}}$, or $\mathbb{R}_{+}^{d_{r, n}}$ in the case of a boundary point.

We need a smooth version of Mostow's slice theorem [Mostow 1957; Bredon 1972]. Let $\mathcal{N}_{x}$ denote a neighborhood at $x$.

Lemma 4.2. For any $[\rho] \in \mathfrak{X}_{r}(K)$, there is a neighborhood $\mathcal{N}_{[\rho]}$ homeomorphic to $H^{1}\left(\mathrm{~F}_{r} ; \mathfrak{k}_{\mathrm{Ad}_{\rho}}\right) / \mathrm{Stab}_{\rho}$. Moreover,

$$
T_{[\rho]} \mathfrak{X}_{r}(K) \cong T_{0}\left(H^{1}\left(\mathrm{~F}_{r} ; \mathfrak{k}_{\mathrm{Ad}_{\rho}}\right) / \operatorname{Stab}_{\rho}\right) .
$$

Proof. Let $\mathfrak{R}_{r}(K)=\operatorname{Hom}\left(\mathrm{F}_{r}, K\right)$. Since $\rho \in \mathfrak{R}_{r}(K)$ is a smooth point, $T_{\rho} \mathfrak{R}_{r}(K) \cong$ $Z^{1}\left(\mathrm{~F}_{r} ; \mathfrak{k}_{\mathrm{Ad}_{\rho}}\right)$. Moreover, $T_{\rho} \mathrm{Orb}_{\rho} \cong B^{1}\left(\mathrm{~F}_{r} ; \mathfrak{k}_{\mathrm{Ad}_{\rho}}\right) \subset Z^{1}\left(\mathrm{~F}_{r} ; \mathfrak{k}_{\mathrm{Ad}_{\rho}}\right)$. Since $\mathrm{Stab}_{\rho}$ is compact and acts on $B^{1}\left(\mathrm{~F}_{r} ; \mathfrak{k}_{\mathrm{Ad}_{\rho}}\right)$, there exists a $\mathrm{Stab}_{\rho}$-invariant complement $W$. Thus $Z^{1}\left(\mathrm{~F}_{r} ; \mathfrak{k}_{\mathrm{Ad}_{\rho}}\right) \cong T_{\rho} \mathfrak{R}_{r}(K) \cong B^{1}\left(\mathrm{~F}_{r} ; \mathfrak{k}_{\mathrm{Ad}_{\rho}}\right) \oplus W$, which respects the action of the stabilizer. Since $\mathfrak{R}_{r}(K)$ is a smooth compact Riemannian manifold we can invariantly exponentiate $W$ to obtain a slice $\exp (W)=S \subset \mathfrak{R}_{r}(K)$ such that $T_{\rho} S=W$. Therefore, $T_{\rho} S \cong H^{1}\left(\mathrm{~F}_{r} ; \mathfrak{k}_{\mathrm{Ad}_{\rho}}\right)$ as $\operatorname{Stab}_{\rho}$-spaces.

Saturating $S$ by $K$ we obtain an open $K$-invariant space, which contains the orbit of $\rho$ since $\rho \in S$; namely $U=K(S)$. Since $U$ is open $T_{\rho} U=T_{\rho} \Re_{r}(K)$, and since it is saturated $U / K \cong S / \operatorname{Stab}_{\rho}$ is an open subset of $\mathfrak{X}_{r}(K)$.

Putting these observations together we conclude $S$ is locally diffeomorphic to $T_{\rho} S$ which implies the neighborhood $U / K \cong H^{1}\left(\mathrm{~F}_{r} ; \mathfrak{k}_{\mathrm{Ad}_{\rho}}\right) / \mathrm{Stab}_{\rho}$, which establishes our first claim.

Then $S / \operatorname{Stab}_{\rho}$ is locally homeomorphic to $T_{\rho} S / \mathrm{Stab}_{\rho}$, which then implies

$$
T_{[\rho]}\left(S / \operatorname{Stab}_{\rho}\right) \cong T_{0}\left(T_{\rho} S / \operatorname{Stab}_{\rho}\right) .
$$

But

$$
T_{[\rho]} \mathfrak{X}_{r}(K)=T_{[\rho]}(U / K) \cong T_{[\rho]}\left(S / \operatorname{Stab}_{\rho}\right)
$$

and

$$
T_{0}\left(T_{\rho} S / \mathrm{Stab}_{\rho}\right) \cong T_{0}\left(H^{1}\left(\mathrm{~F}_{r} ; \mathfrak{k}_{\mathrm{Ad}_{\rho}}\right) / \mathrm{Stab}_{\rho}\right) .
$$

Equations (7), (8), and (9) together complete the proof. 
Remark 4.3. The above lemma holds for all compact Lie groups $K$.

Theorem 4.4. Let $r, n \geq 2$ and let $\rho \in \mathfrak{R}_{r}\left(\mathrm{SU}_{n}\right)$ be of reduced type $\left[n_{1}, n_{2}\right]$. Then, there exists a neighborhood $[\rho] \in \mathcal{N} \subset \mathfrak{X}_{r}\left(\mathrm{SU}_{n}\right)$ that is homeomorphic to $\mathbb{R}^{d_{S U_{n}}} \times$ $\mathscr{C}\left(\mathbb{C P}{ }^{(r-1) n_{1} n_{2}-1}\right)$, where $d_{\mathrm{SU}_{n}}=(r-1)\left(n_{1}^{2}+n_{2}^{2}-1\right)+1$. Also, if $\rho \in \mathfrak{R}_{r}\left(\mathrm{U}_{n}\right)$ is of reduced type $\left[n_{1}, n_{2}\right]$, there exists a neighborhood $[\rho] \in \mathcal{N} \subset \mathfrak{X}_{r}\left(\mathrm{U}_{n}\right)$ that is homeomorphic to $\mathbb{R}^{d_{\cup_{n}}} \times \mathscr{C}\left(\mathbb{C} P^{(r-1) n_{1} n_{2}-1}\right)$, where $d_{\cup_{n}}=(r-1)\left(n_{1}^{2}+n_{2}^{2}\right)+2$.

Corollary 4.5. If $K=\mathrm{U}_{n}$ or $K=\mathrm{SU}_{n}$, both $r, n \geq 2$, and $(r, n) \neq(2,2),(2,3)$, or $(3,2)$, then $\mathfrak{X}_{r}(K)$ is not a manifold with boundary.

Proof. Theorem 4.4 implies that $\mathfrak{X}_{r}\left(\mathrm{U}_{n}\right)$ and $\mathfrak{X}_{r}\left(\mathrm{SU}_{n}\right)$ are manifolds only if $\mathbb{R}^{d_{\cup_{n}}} \times$ $\mathscr{C}\left(\mathbb{C P}^{(r-1) n_{1} n_{2}-1}\right)$ and $\mathbb{R}^{d_{\mathrm{S}} \cup_{n}} \times \mathscr{C}\left(\mathbb{C P}^{(r-1) n_{1} n_{2}-1}\right)$, respectively, are locally Euclidean. By Lemma 4.1, this can only be the case if $n_{1} n_{2}(r-1)-1 \in\{0,1\}$, with $n=n_{1}+n_{2}$ and $n_{1}, n_{2}>0$. In the first case, $n_{1} n_{2}(r-1)=1$, which implies $n_{1}=n_{2}=1$ and $r=2$, so $(r, n)=(2,2)$. From Section $2 \mathrm{~B}$ we know $\mathfrak{X}_{2}\left(\mathrm{U}_{2}\right)$ and $\mathfrak{X}_{2}\left(\mathrm{SU}_{2}\right)$ are manifolds with boundary, and we conclude the neighborhood in this case is half-Euclidean since $\mathcal{N}=\mathbb{R}^{d} \times[0,1)$, for appropriate $d$.

The other possibility is $n_{1} n_{2}(r-1)=2$ so that $n_{1}=2$ and $n_{2}=1$, or $n_{1}=1$ and $n_{2}=2$, and $r=2$. This is the case $(r, n)=(2,3)$. Otherwise, $r=3$ and $n_{1}=n_{2}=1$, which is the case $(r, n)=(3,2)$. Moreover, from Section 2B these two are the only cases which are manifolds.

Having exhausted all possibilities, the proof is complete.

We now prove Theorem 4.4.

Proof of Theorem 4.4. Similar to Theorem 3.21, there is a direct computational proof of Theorem 4.4. However, using Theorem 3.21, Lemma 3.19 and the relation between $K$ and its complexification, we can provide a shorter argument.

Let $\tau$ be the Cartan involution on $\mathfrak{g}=\mathfrak{g l}(n, \mathbb{C})$, the Lie algebra of $\mathrm{GL}_{n}$, which is just the linear map $A \mapsto-\bar{A}^{T}$, acting on a matrix $A \in \mathfrak{g l}(n, \mathbb{C})$. By definition, the fixed point subspace of $\tau$ is $\mathfrak{k}$, the Lie algebra $\mathfrak{u}_{n}$ of $\bigcup_{n}$. One easily checks that $\tau$ induces an involution on $Z^{1}\left(\mathrm{~F}_{r} ; \mathfrak{g}_{\mathrm{Ad}_{\rho}}\right) \cong \mathfrak{g}^{r}$, whose fixed subspace is $Z^{1}\left(\mathrm{~F}_{r} ; \mathfrak{k}_{\mathrm{Ad}_{\rho}}\right) \cong \mathfrak{k}^{r}$, and similarly $B^{1}\left(\mathrm{~F}_{r} ; \mathfrak{g}_{\mathrm{Ad}_{\rho}}\right)^{\tau}=B^{1}\left(\mathrm{~F}_{r} ; \mathfrak{k}_{\mathrm{Ad}_{\rho}}\right)$. This implies that $\tau$ induces an involution, also denoted $\tau$, on the first cohomology, and that $H^{1}\left(\mathrm{~F}_{r} ; \mathfrak{k}_{\mathrm{Ad}_{\rho}}\right)$ is naturally isomorphic to $H^{1}\left(\mathrm{~F}_{r} ; \mathfrak{g}_{\mathrm{Ad}_{\rho}}\right)^{\tau}$.

Now, assume that $\rho=\rho_{1} \oplus \rho_{2} \in U_{r, n} \cap \Re_{r}\left(\mathrm{U}_{n}\right)$, is of reduced type $\left[n_{1}, n_{2}\right]$ $\left(n_{1}, n_{2}>0, n_{1}+n_{2}=n\right)$. Note that $\rho_{1}$ and $\rho_{2}$ are irreducible representations in $\mathfrak{R}_{r}\left(\mathrm{U}_{n_{1}}\right)$ and $\mathfrak{R}_{r}\left(\mathrm{U}_{n_{2}}\right)$, respectively, and with respect to the $\mathrm{PU}_{n}$ conjugation action $\operatorname{Stab}_{\rho} \cong S^{1}$. Then a cocycle $\phi \in Z^{1}\left(\mathrm{~F}_{r} ; \mathfrak{k}_{\mathrm{Ad}_{\rho}}\right) \cong \mathfrak{k}^{r}$ has the form

$$
\phi=\left(\begin{array}{rr}
\phi_{1} & A \\
-\bar{A}^{T} & \phi_{2}
\end{array}\right)
$$


where $\phi_{i} \in Z^{1}\left(\mathrm{~F}_{r} ; \mathfrak{k}_{\mathrm{Ad}_{\rho_{i}}}\right)$, and as in Theorem 3.21, $A$ is now an arbitrary $r$-tuple of $n_{1} \times n_{2}$ matrices. This shows that $\tau$ respects the decomposition in Equation (6), so we get

$$
\begin{aligned}
H^{1}\left(\mathrm{~F}_{r} ; \mathfrak{k}_{\mathrm{Ad}_{\rho}}\right)=H^{1}\left(\mathrm{~F}_{r} ; \mathfrak{g}_{\mathrm{Ad}_{\rho}}\right)^{\tau} & =H^{1}\left(\mathrm{~F}_{r} ; \mathfrak{g}_{\mathrm{Ad}_{\rho_{1}}}\right)^{\tau} \oplus H^{1}\left(\mathrm{~F}_{r} ; \mathfrak{g}_{\mathrm{Ad}_{\rho_{2}}}\right)^{\tau} \oplus W^{\tau} \\
& =H^{1}\left(\mathrm{~F}_{r} ; \mathfrak{k}_{\mathrm{Ad}_{\rho_{1}}}\right) \oplus H^{1}\left(\mathrm{~F}_{r} ; \mathfrak{k}_{\mathrm{Ad}_{\rho_{2}}}\right) \oplus F
\end{aligned}
$$

where, by the form of the cocycles above, we can write

$$
F:=W^{\tau}=\left\{(z,-\bar{z}): z \in \mathbb{C}^{n_{1} n_{2}(r-1)}\right\} ;
$$

using also $\operatorname{dim}_{\mathbb{C}} W=2 n_{1} n_{2}(r-1)$.

It follows from Lemma 4.2 that a neighborhood of $\rho$ is locally homeomorphic to $H^{1}\left(\mathrm{~F}_{r} ; \mathfrak{k}_{\mathrm{Ad}_{\rho}}\right) / \mathrm{Stab}_{\rho}$. As in the proof of Theorem 3.21, the action of $\mathrm{Stab}_{\rho}=S^{1}$ does not affect $H^{1}\left(\mathrm{~F}_{r} ; \mathfrak{k}_{\mathrm{Ad}_{\rho_{i}}}\right), i=1,2$, and we conclude that

$$
\begin{aligned}
& H^{1}\left(\mathrm{~F}_{r} ; \mathfrak{k}_{\mathrm{Ad}_{\rho}}\right) / \operatorname{Stab}_{\rho}=H^{1}\left(\mathrm{~F}_{r} ; \mathfrak{k}_{\mathrm{Ad}_{\rho_{1}}}\right) \oplus H^{1}\left(\mathrm{~F}_{r} ; \mathfrak{k}_{\mathrm{Ad}_{\rho_{2}}}\right) \oplus F / S^{1} \\
& \cong \mathbb{R}^{d_{\cup_{n}}} \oplus \mathscr{C}\left(\mathbb{C P}^{n_{1} n_{2}(r-1)-1}\right),
\end{aligned}
$$

by using Lemma 3.19. The dimension $d_{\cup_{n}}$ is computed by:

$$
d_{\mathrm{U}_{n}}=\sum_{i=1}^{2} \operatorname{dim}_{\mathbb{R}} H^{1}\left(\mathrm{~F}_{r} ; \mathfrak{k}_{\mathrm{Ad}_{\rho_{i}}}\right)=\left(n_{1}^{2}+n_{2}^{2}\right)(r-1)+2 .
$$

The case of $K=\mathrm{SU}_{n}$ is similar.

Remark 4.6. In [Le Bruyn and Teranishi 1990] it is shown that the cases $(2,2)$, $(2,3)$, and $(3,2)$ are also the only examples which are complete intersections.

Remark 4.7. Note that using the identity representation (maximal stabilizer) results in $H^{1}\left(\mathrm{~F}_{r} ; \mathfrak{k}_{\mathrm{Ad}_{\mathrm{id}}}\right) / \mathrm{Stab}_{\mathrm{id}}=\mathfrak{k}^{r} / \mathrm{SU}_{n}$ since the coboundaries are trivial. Removing a point results in a homological sphere quotient $S^{\left(n^{2}-1\right)(r-1)-1} / \mathrm{SU}_{n}$. If there was a Euclidean neighborhood about the identity, then this sphere quotient would be a homology sphere $S^{\left(n^{2}-1\right)(r-2)-1}$. We find this quite likely to give a different obstruction. At the other extreme (central stabilizer) the points are smooth and thus admit Euclidean neighborhoods.

Conjecture 4.8. If $K$ is equal to $\mathrm{SU}_{n}$ or $\mathrm{U}_{n},[\rho] \in \mathfrak{X}_{r}(K)^{\mathrm{red}}, r, n \geq 2$, and $(r, n) \neq$ $(2,2),(2,3)$ or $(3,2)$, then there does not exists a neighborhood of $[\rho]$ that is Euclidean.

We proved this conjecture for representations of reduced type $\left[n_{1}, n_{2}\right]$ above. In fact, it seems likely that the neighborhoods around most singularities do not even admit an orbifold structure (not homeomorphic to a finite quotient of a Euclidean ball). 
4B. $\mathfrak{X}_{r}\left(\mathrm{SL}_{n}\right)$ and $\mathfrak{X}_{r}\left(\mathrm{GL}_{n}\right)$. In this last subsection, we complete the proof of Theorem 1.2 by proving the following result.

Theorem 4.9. Let $r, n \geq 2$ and let $G$ be $\mathrm{SL}_{n}$ or $\mathrm{GL}_{n}$. $\mathfrak{X}_{r}(G)$ is a topological manifold with boundary if and only if $(r, n)=(2,2)$.

Proof. By Remark 3.17, $H^{1}\left(\mathrm{~F}_{r} ; \mathfrak{g}_{\mathrm{Ad}_{\rho}{ }_{\rho \mathrm{s}}}\right) / / \mathrm{Stab}_{\rho^{\mathrm{ss}}}$ is an étale neighborhood; that is, an algebraic set that maps, via an étale mapping, to an open set (in the ball topology) of $\mathfrak{X}_{r}(G)$. Thus we see that at a reducible representation with minimal stabilizer ( $\mathbb{C}^{*}$ for $\mathrm{SL}_{n}$ and $\mathbb{C}^{*} \times \mathbb{C}^{*}$ for $\mathrm{GL}_{n}$ ), that this neighborhood is étale equivalent to $\mathbb{C}^{\left(n_{1}^{2}+n_{2}^{2}\right)(r-1)+2} \times \mathscr{C}\left(\mathbb{C} \mathrm{P}^{(r-1) n_{1} n_{2}-1} \times \mathbb{C} \mathrm{P}^{(r-1) n_{1} n_{2}-1}\right)$ in $\mathfrak{X}_{r}\left(\mathrm{GL}_{n}\right)$, where the cone here is the affine cone defined over $\mathbb{C}^{*}$. In $\mathfrak{X}_{r}\left(\mathrm{SL}_{n}\right)$ we have a similar neighborhood. Either way, these spaces are not locally Euclidean neighborhoods for $r, n \geq 2$ unless $n=2=r$ which implies that $n_{1}=1=n_{2}$. This is seen by similar arguments given above in the compact cases.

\section{Acknowledgements}

The second author thanks W. Goldman for helpful discussions, the Instituto Superior Técnico for its support during the 2007-2009 academic years, and A. Sikora for useful conversations. We also thank M. Reineke and O. Serman for pointing out relevant references.

\section{References}

[Bochnak et al. 1998] J. Bochnak, M. Coste, and M.-F. Roy, Real algebraic geometry, Ergebnisse der Mathematik und ihrer Grenzgebiete (3) 36, Springer, Berlin, 1998. MR 2000a:14067 Zbl 0912.14023

[Bratholdt and Cooper 2001] S. Bratholdt and D. Cooper, "On the topology of the character variety of a free group", Rend. Istit. Mat. Univ. Trieste 32:Suppl. 1 (2001), 45-53. MR 2003d:14072 Zbl 1062.14505

[Bredon 1972] G. E. Bredon, Introduction to compact transformation groups, Pure and Applied Mathematics 46, Academic Press, New York, 1972. MR 54 \#1265 Zbl 0246.57017

[Dolgachev 2003] I. Dolgachev, Lectures on invariant theory, London Mathematical Society Lecture Note Series 296, Cambridge University Press, Cambridge, 2003. MR 2004g:14051 Zbl 1023.13006

[Drézet 2004] J.-M. Drézet, "Luna's slice theorem and applications", pp. 39-89 in Algebraic group actions and quotients (Wykno, 2000), edited by J. Wiśniewski, Hindawi, Cairo, 2004. MR 2006k:14082 Zbl 1109.14307

[Florentino and Lawton 2009] C. Florentino and S. Lawton, "The topology of moduli spaces of free group representations”, Math. Ann. 345:2 (2009), 453-489. MR 2010h:14075 Zbl 1200.14093

[Fricke and Klein 1912] R. Fricke and F. Klein, Vorlesungen über die Theorie der automorphen Funktionen, II: Die funktionentheoretischen Ausführungen und die Andwendungen, 3: Direkte Beweismethoden der Fundamentaltheoreme und Anhang, Teubner, Leipzig, 1912. Reprinted in Bibliotheca Mathematica Teubneriana, vol. 4, by Johnson Reprint, New York, 1965. MR 32 \#1348 JFM 43.0529.08 
[Goldman 1984] W. M. Goldman, "The symplectic nature of fundamental groups of surfaces", Adv. in Math. 54:2 (1984), 200-225. MR 86i:32042 Zbl 0574.32032

[Goldman 1990] W. M. Goldman, "Convex real projective structures on compact surfaces", J. Differential Geom. 31:3 (1990), 791-845. MR 91b:57001 Zbl 0711.53033

[Goldman 2009] W. M. Goldman, "Trace coordinates on Fricke spaces of some simple hyperbolic surfaces", pp. 611-684 in Handbook of Teichmüller theory, II, edited by A. Papadopoulos, IRMA Lect. Math. Theor. Phys. 13, Eur. Math. Soc., Zürich, 2009. MR 2010j:30093 Zbl 1175.30043

[Harris 1992] J. Harris, Algebraic geometry: a first course, Graduate Texts in Mathematics 133, Springer, New York, 1992. MR 93j:14001 Zbl 0779.14001

[Heusener and Porti 2004] M. Heusener and J. Porti, "The variety of characters in PSL 2 (C)", Bol. Soc. Mat. Mexicana (3) 10:Special Issue (2004), 221-237. MR 2006m:57020 Zbl 1100.57014

[Johnson and Millson 1987] D. Johnson and J. J. Millson, "Deformation spaces associated to compact hyperbolic manifolds", pp. 48-106 in Discrete groups in geometry and analysis (New Haven, CT, 1986), edited by R. Howe, Progr. Math. 67, Birkhäuser, Boston, 1987. MR 88j:22010 Zbl 0664.53023

[Lang 2002] S. Lang, Algebra, 3rd ed., Graduate Texts in Mathematics 211, Springer, New York, 2002. MR 2003e:00003 Zbl 0984.00001

[Laszlo 1996] Y. Laszlo, "Local structure of the moduli space of vector bundles over curves", Comment. Math. Helv. 71:3 (1996), 373-401. MR 97j:14012 Zbl 0949.14015

[Lawton 2007] S. Lawton, "Generators, relations and symmetries in pairs of $3 \times 3$ unimodular matrices”, J. Algebra 313:2 (2007), 782-801. MR 2008k:16039 Zbl 1119.13004

[Le Bruyn and Procesi 1987] L. Le Bruyn and C. Procesi, "Étale local structure of matrix invariants and concomitants", pp. 143-175 in Algebraic groups (Utrecht, 1986), edited by A. M. Cohen et al., Lecture Notes in Math. 1271, Springer, Berlin, 1987. MR 89b:16042 Zbl 0634.14034

[Le Bruyn and Teranishi 1990] L. Le Bruyn and Y. Teranishi, "Matrix invariants and complete intersections”, Glasgow Math. J. 32:2 (1990), 227-229. MR 91d:15059 Zbl 0714.16016

[Luna 1973] D. Luna, "Slices étales”, pp. 81-105 in Sur les groupes algébriques, Bull. Soc. Math. France Mém. 33, Soc. Math. France, Paris, 1973. MR 49 \#7269 Zbl 0286.14014

[Mostow 1957] G. D. Mostow, "Equivariant embeddings in Euclidean space", Ann. of Math. (2) 65 (1957), 432-446. MR 19,291c Zbl 0080.16701

[Mukai 2003] S. Mukai, An introduction to invariants and moduli, Cambridge Studies in Advanced Mathematics 81, Cambridge University Press, Cambridge, 2003. MR 2004g:14002 Zbl 1033.14008

[Narasimhan and Ramanan 1969] M. S. Narasimhan and S. Ramanan, "Moduli of vector bundles on a compact Riemann surface", Ann. of Math. (2) 89 (1969), 14-51. MR 39 \#3518 Zbl 0186.54902

[Narasimhan and Seshadri 1965] M. S. Narasimhan and C. S. Seshadri, "Stable and unitary vector bundles on a compact Riemann surface", Ann. of Math. (2) 82 (1965), 540-567. MR 32 \#1725 Zbl 0171.04803

[Procesi and Schwarz 1985] C. Procesi and G. Schwarz, "Inequalities defining orbit spaces", Invent. Math. 81:3 (1985), 539-554. MR 87h:20078 Zbl 0578.14010

[Schwarz 2004] G. W. Schwarz, "Group actions and quotients for compact Lie groups and algebraic groups", pp. 209-227 in Invariant theory in all characteristics (Kingston, ON, 2002), edited by H. E. A. E. Campbell and D. L. Wehlau, CRM Proc. Lecture Notes 35, Amer. Math. Soc., Providence, RI, 2004. MR 2005d:20083 Zbl 1131.14312

[Shafarevich 1994] I. R. Shafarevich, Basic algebraic geometry, 2: Schemes and complex manifolds, 2nd ed., Springer, Berlin, 1994. MR 95m:14002 Zbl 0797.14002 
[Sikora 2012] A. S. Sikora, "Character varieties", Trans. Amer. Math. Soc. 364:10 (2012), 5173-5208. MR 2931326 arXiv 0902.2589

[Sullivan 1971] D. Sullivan, "Combinatorial invariants of analytic spaces", pp. 165-168 in Singularities - Symposium I (Liverpool, 1969-1970), edited by C. T. C. Hall, Lecture Notes in Math. 192, Springer, Berlin, 1971. MR 43 \#4063 Zbl 0227.32005

[Vogt 1889] H. Vogt, "Sur les invariants fondamentaux des équations différentielles linéaires du second ordre”, Ann. Sci. École Norm. Sup. (3) 6 (1889), 3-71. MR 1508833 JFM 21.0314.01

[Weil 1964] A. Weil, "Remarks on the cohomology of groups", Ann. of Math. (2) 80 (1964), 149-157. MR 30 \#199 Zbl 0192.12802

Received May 30, 2012.

CARLOS Florentino

Departamento de MATEMÁtica

INSTITUTO SUPERIOR TÉCNICO

1049-001 LISBOA

PORTUGAL

carlos.florentino@math.ist.utl.pt

SEAN LAWTON

DEPARTMENT OF MATHEMATICS

UNIVERSITY OF TEXAS-PAN AMERICAN

1201 WEST UNIVERSITY DRIVE

EDINBURG, TX 78539

UNITED STATES

lawtonsd@utpa.edu 


\title{
PACIFIC JOURNAL OF MATHEMATICS
}

\author{
http://pacificmath.org \\ Founded in 1951 by \\ E. F. Beckenbach (1906-1982) and F. Wolf (1904-1989)
}

\section{EDITORS}

V. S. Varadarajan (Managing Editor)

Department of Mathematics

University of California

Los Angeles, CA 90095-1555

pacific@math.ucla.edu

Vyjayanthi Chari

Department of Mathematics

University of California

Riverside, CA 92521-0135

chari@math.ucr.edu

\section{Robert Finn}

Department of Mathematics Stanford University

Stanford, CA 94305-2125

finn@math.stanford.edu

Kefeng Liu

Department of Mathematics

University of California

Los Angeles, CA 90095-1555

liu@math.ucla.edu
Darren Long

Department of Mathematics

University of California

Santa Barbara, CA 93106-3080

long@math.ucsb.edu

Jiang-Hua Lu

Department of Mathematics

The University of Hong Kong

Pokfulam Rd., Hong Kong jhlu@maths.hku.hk

Alexander Merkurjev

Department of Mathematics

University of California

Los Angeles, CA 90095-1555

merkurev@math.ucla.edu
Sorin Popa

Department of Mathematics University of California

Los Angeles, CA 90095-1555 popa@math.ucla.edu

Jie Qing

Department of Mathematics

University of California

Santa Cruz, CA 95064

qing@cats.ucsc.edu

Jonathan Rogawski

Department of Mathematics

University of California

Los Angeles, CA 90095-1555

jonr@math.ucla.edu

\section{PRODUCTION}

pacific@math.berkeley.edu

\section{SUPPORTING INSTITUTIONS}

ACADEMIA SINICA, TAIPEI

CALIFORNIA INST. OF TECHNOLOGY INST. DE MATEMÁTICA PURA E APLICADA KEIO UNIVERSITY

MATH. SCIENCES RESEARCH INSTITUTE NEW MEXICO STATE UNIV.

OREGON STATE UNIV.

\author{
STANFORD UNIVERSITY \\ UNIV. OF BRITISH COLUMBIA \\ UNIV. OF CALIFORNIA, BERKELEY \\ UNIV. OF CALIFORNIA, DAVIS \\ UNIV. OF CALIFORNIA, LOS ANGELES \\ UNIV. OF CALIFORNIA, RIVERSIDE \\ UNIV. OF CALIFORNIA, SAN DIEGO \\ UNIV. OF CALIF., SANTA BARBARA
}

\author{
UNIV. OF CALIF., SANTA CRUZ \\ UNIV. OF MONTANA \\ UNIV. OF OREGON \\ UNIV. OF SOUTHERN CALIFORNIA \\ UNIV. OF UTAH \\ UNIV. OF WASHINGTON \\ WASHINGTON STATE UNIVERSITY
}

These supporting institutions contribute to the cost of publication of this Journal, but they are not owners or publishers and have no responsibility for its contents or policies.

See inside back cover or pacificmath.org for submission instructions.

The subscription price for 2012 is US \$420/year for the electronic version, and \$485/year for print and electronic.

Subscriptions, requests for back issues from the last three years and changes of subscribers address should be sent to Pacific Journal of Mathematics, P.O. Box 4163, Berkeley, CA 94704-0163, U.S.A. Prior back issues are obtainable from Periodicals Service Company, 11 Main Street, Germantown, NY 12526-5635. The Pacific Journal of Mathematics is indexed by Mathematical Reviews, Zentralblatt MATH, PASCAL CNRS Index, Referativnyi Zhurnal, Current Mathematical Publications and the Science Citation Index.

The Pacific Journal of Mathematics (ISSN 0030-8730) at the University of California, c/o Department of Mathematics, 969 Evans Hall, Berkeley, CA 94720-3840, is published monthly except July and August. Periodical rate postage paid at Berkeley, CA 94704, and additional mailing offices. POSTMASTER: send address changes to Pacific Journal of Mathematics, P.O. Box 4163, Berkeley, CA 94704-0163.

PJM peer review and production are managed by EditFLOW ${ }^{\mathrm{TM}}$ from Mathematical Sciences Publishers.

PUBLISHED BY PACIFIC JOURNAL OF MATHEMATICS

at the University of California, Berkeley 94720-3840

A NON-PROFIT CORPORATION

Typeset in LATEX

Copyright $(02012$ by Pacific Journal of Mathematics 


\section{PACIFIC JOURNAL OF MATHEMATICS}

Volume $260 \quad$ No. $1 \quad$ November 2012

The decomposition of global conformal invariants: Some technical proofs II

SPYROS ALEXAKIS

On deformation quantizations of hypertoric varieties

GWYN BELLAMY and TOSHIRO KUWABARA

Almost factoriality of integral domains and Krull-like domains

GYU Whan CHANG, HWANKOO KIM and JUNG WOOK LIM

Singularities of free group character varieties

CARLOS FLORENTINO and SEAN LAWTON

Energy identity for the maps from a surface with tension field bounded 181 in $L^{p}$

LI JIAYU and ZHU XIANGRONG

Remarks on some isoperimetric properties of the $k-1$ flow

YU-CHU LIN and DONG-Ho TSAI

Demystifying a divisibility property of the Kostant partition function 215

KAROLA MÉSZÁros

Exceptional Lie algebras, SU(3), and Jordan pairs

PIERO TRUINI

Lower estimate of Milnor number and characterization of isolated homogeneous hypersurface singularities

Stephen S.-T. Yau and HuAiqing Zuo 